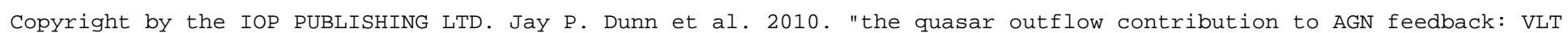
measurements of SDSS J0318-0600," ApJ 709611 doi:10.1088/0004-637X/709/2/611

The Astrophysical Journal, 709:611-631, 2010 February 1

(C) 2010. The American Astronomical Society. All rights reserved. Printed in the U.S.A.

\title{
THE QUASAR OUTFLOW CONTRIBUTION TO AGN FEEDBACK: VLT MEASUREMENTS OF
} SDSS J0318-0600*

\author{
Jay P. Dunn ${ }^{1}$, Manuel Bautista ${ }^{1,2}$, Nahum Arav $^{1},{\text { Max } \text { Moe }^{3,4}, \text { Kirk Korista }^{2} \text {, Elisa Costantini }}^{5}$, Chris Benn ${ }^{6}$, \\ SARA Ellison ${ }^{7}$, AND Doug EdMONDS ${ }^{1}$ \\ ${ }^{1}$ Department of Physics, Virginia Tech, Blacksburg, VA 24061, USA; jdunn77@vt.edu, arav@ vt.edu, edmonds@ vt.edu \\ 2 Department of Physics, Western Michigan University, Kalamazoo, MI 49008-5252, USA; kirk.korista@wmich.edu, manuel.bautista@wmich.edu \\ ${ }^{3}$ Department of Astrophysical and Planetary Sciences and Center for Astrophysics and Space Astronomy, University of Colorado, 389-UCB, \\ Boulder, CO 80309, USA \\ ${ }^{4}$ Harvard-Smithsonian Center for Astrophysics, Cambridge, MA 02138, USA; mmoe@cfa.harvard.edu \\ ${ }^{5}$ SRON National Institute for Space Research, Sorbonnelaan 2, 3584 CA Utrecht, The Netherlands \\ ${ }^{6}$ Isaac Newton Group, Apartado 321, E-38700 Santa Cruz de La Palma, Spain \\ ${ }^{7}$ Department of Physics and Astronomy, University of Victoria, Victoria, BC V8P 1A1, Canada \\ Received 2009 February 26; accepted 2009 December 2; published 2010 January 6
}

\begin{abstract}
We present high spectral resolution Very Large Telescope observations of the broad absorption line quasar SDSS J0318-0600. This high-quality data set allows us to extract accurate ionic column densities and determine an electron number density of $n_{e}=10^{3.3 \pm 0.2 \mathrm{~cm}^{-3}}$ for the main outflow absorption component. The heavily reddened spectrum of SDSS J0318-0600 requires purely silicate dust with a reddening curve characteristic of predominately large grains, from which we estimate the bolometric luminosity. We carry out photoionization modeling to determine the total column density, ionization parameter, and distance of the gas and find that the photoionization models suggest abundances greater than solar. Due to the uncertainty in the location of the dust extinction, we arrive at two viable distances for the main ouflow component from the central source, 6 and $17 \mathrm{kpc}$, where we consider the $6 \mathrm{kpc}$ location as somewhat more physically plausible. Assuming the canonical global covering of $20 \%$ for the outflow and a distance of $6 \mathrm{kpc}$, our analysis yields a mass flux of $120 M_{\odot} \mathrm{yr}^{-1}$ and a kinetic luminosity that is $\sim 0.1 \%$ of the bolometric luminosity of the object. Should the dust be part of the outflow, then these values are $\sim 4 \times$ larger. The large mass flux and kinetic luminosity make this outflow a significant contributor to active galactic nucleus feedback processes.
\end{abstract}

Key words: quasars: absorption lines - quasars: individual (SDSS J0318-0600)

Online-only material: color figure, figure set, machine readable table

\section{INTRODUCTION}

Quasar outflows, seen as ultraviolet blueshifted absorption features in their spectra, may contribute to a variety of active galactic nucleus (AGN) feedback mechanisms (Elvis 2006). Such feedback processes are invoked to explain the evolution of the super massive black hole (SMBH; Silk \& Rees 1998; Blandford \& Begelman 1999), evolution of the host galaxy (Di Matteo et al. 2005), shape of the observed quasar luminosity function (Hopkins et al. 2005a; Springel et al. 2005; Menci et al. 2006), and the enrichment of the intra-cluster and inter-galactic media (Moll et al. 2007).

The contribution of quasar outflows to AGN feedback depends mainly on their mass flux $(\dot{M})$ and kinetic luminosity $\left(\dot{E}_{k}\right)$, both of which depend linearly on the total hydrogen column density $\left(N_{H}\right)$ of the outflow and its characteristic distance $(R)$ from the central source (see Equations (9) and (11) in Section 6). Until recently, only loosely constrained estimates have been available for $N_{H}$ and $R$ in the same outflow. Morris et al. (1986) and Tripp et al. (1996) were only able to find lower limits for the distance to outflows in GC 1556+335 and HS 1946+7658, respectively. Wampler et al. (1995), analyzing the spectrum of QSO 0059-2735, constrained the distance of the outflow to a range spanning over an order of magnitude.

\footnotetext{
* Based on observations collected at the European Organization for Astronomical Research in the Southern Hemisphere, Chile; under program 078.B-0433.
}

de Kool et al. (2001) determined the distance to the outflow observed in FIRST J1044+3656 to within a factor of 100 and had a similar uncertainty in the inferred $N_{H}$ (see their Table 3), which led to an uncertainty in $\dot{M}$ and $\dot{E}_{k}$ of approximately 1 order of magnitude. In the cases of FIRST J1214+2803 \& FIRST J0840+3633, de Kool et al. (2002a, 2002b) were able to constrain $\dot{M}$ and $\dot{E}_{k}$ to a similar accuracy. For the quasar 3C 191, Hamann et al. (2001) estimated $\dot{M}$ and $\dot{E}_{k}$ to within a factor of a few. However, beside their internal uncertainties, all the works cited above suffered from unquantified systematic errors due to the use of inadequate absorption models that led to unreliable measurements of column densities in the observed troughs, which are crucial for determining almost every physical aspect of the outflows: the ionization equilibrium and abundances, number density, distance, mass flux, and kinetic luminosity.

In order to determine accurate $\dot{M}$ and $\dot{E}_{k}$ in quasar outflows, we launched a multistage research program: we first developed analysis techniques that allow us to determine reliable trough column densities (Arav et al. 1999a, 1999b, 2002, 2005, 2008; Scott et al. 2004b; Gabel et al. 2005). This was followed by identifying outflow targets in which we can find diagnostics for both $N_{H}$ and $R$. We searched through $\sim 50,000$ QSOs with $z>0.25$ and $r<19.3$ in the Sloan Digital Sky Survey (SDSS) Data Release 6 (Adelman-McCarthy et al. 2008), 100 objects were found to exhibit intrinsic outflow absorption systems containing troughs from excited or metastable levels of one or more of the following ions: He I, Si II, Fe II, Fe III, or Ni II. The key spectral 
features to obtain distances and total column densities for outflows are (1) absorption troughs of excited and metastable levels from which we can determine the electron density and (2) the availability of troughs from many ions, including lines from multiple ions of the same element. We then began observing these objects with a combination of high spectral resolution, large spectral coverage, and high signal-to-noise ratios that in aggregate allows for the use of the analysis techniques mentioned above. Arav et al. (2008) and Korista et al. (2008) used this analysis method on VLT observations of QSO 2359-1241, deriving $\mathrm{N}_{H}$ and hydrogen number density determinations with accuracies better than $25 \%$ and $20 \%$, respectively.

The object analyzed here is SDSS J0318-0600, which was found in our search through the SDSS database (although first discovered by Hall et al. 2002). We chose this object for a high resolution follow-up observation, due to its high apparent brightness and its suitability for VLT observations. SDSS $\mathrm{J} 0318-0600$ is a luminous quasar $\left(\log L_{\mathrm{Bol}} \approx 47.7 \mathrm{erg} \mathrm{s}^{-1}\right.$, see Section 5; $z=1.9668$; Hall et al. 2002; Schneider et al. 2003) that shows a myriad of outflow components ranging from a high-ionization broad absorption line system to several narrow absorption line (NAL) features spanning a velocity range from -2800 to $-7500 \mathrm{~km} \mathrm{~s}^{-1}$, with respect to the quasar's rest frame. In a survey by Hall et al. (2002), it was classified as an iron lowionization broad absorption line system (FeLoBAL) and listed as an unusual quasar due to the BAL located at $\sim 27,000 \mathrm{~km} \mathrm{~s}^{-1}$ and the system of NALs, which appears as a BAL in higher ionization lines such as C IV. They noted that the Fe II UV1, UV2, and UV3 multiplets were detected in absorption and that the UV38 multiplet appeared to be present as well. It was also noted that SDSS J0318-0600 has a heavily reddened spectrum. Prochter et al. (2006) listed this object as a strong Mg II absorber with a rest-frame absorption width $>1.4 \AA$, in a search of Mg II absorbing objects. Herbert-Fort et al. (2006) showed that the object's absorption was strong, particularly in Si II $\lambda 1808$, and labeled the absorber as a damped Lyman alpha system. However, in our VLT data, we detect absorption from excited ionic energy levels that conclusively classify the system as an outflow (Hamann et al. 1997).

The outline for this paper is as follows. In Section 2, we present the VLT eschelle observations and their reduction. In Section 3, we discuss our methods of determining and measuring individual kinematic components. We then concentrate on a physical analysis of the main component of the outflow: in Section 4, we present our photoionization analysis of the spectrum and determine the hydrogen column density $\left(N_{H}\right)$, electron density $\left(n_{e}\right)$, distance $(R)$, and temperature $(T)$ for the main absorption component. We derive the mass flux and kinetic luminosity in Section 5, and in Section 6 we discuss our results.

\section{OBSERVATIONS AND REDUCTION}

During 2006 October, we obtained eight echelle spectra, each with an exposure time of $2850 \mathrm{~s}$, of the object SDSS J0318-0600 $(z=1.967$, Schneider et al. 2007, R.A. 03:18:56.7, decl. -06:00:37.4) with the VLT. The observations were carried out by European Southern Observatory (ESO) staff and we present the observational parameters in Table 1 . The spectra were taken using the UV-Visual Echelle Spectrograph (UVES) with a slit width of 1.0 arcsec, and a central wavelength position of 5800 and $3460 \AA$ for the red (Grating/Filter $=$ CD1/ HER5) and blue (Grating/Filter $=$ CD3/SHP700) detectors respectively. A slit width of 1.0 arcsec gives a resolving power of
Table 1

VLT Observations of QSO J0318-0600

\begin{tabular}{ccccc}
\hline \hline Date & Time $^{\mathrm{a}}$ & JD $^{\mathrm{b}}$ & Airmass & Seeing \\
\hline 2006 Sep 30 & $04: 35: 01$ & 54008.19092 & 1.41 & 1.08 \\
2006 Oct 01 & $03: 51: 30$ & 54009.25268 & 1.12 & 1.07 \\
2006 Oct 01 & $06: 53: 51$ & 54009.28740 & 1.06 & 0.97 \\
2006 Oct 15 & $05: 55: 23$ & 54023.24679 & 1.06 & 1.00 \\
2006 Oct 19 & $05: 34: 22$ & 54027.23220 & 1.07 & 0.82 \\
2006 Oct 20 & $06: 49: 21$ & 54028.28427 & 1.07 & 0.71 \\
2006 Oct 22 & $04: 45: 58$ & 54030.20063 & 1.10 & 0.66 \\
2006 Oct 22 & $05: 38: 28$ & 54030.23505 & 1.06 & 0.55 \\
\hline
\end{tabular}

Notes.

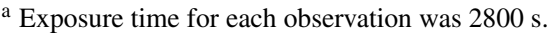

b Julian Date presented in days +2400000 .

$\sim 40,000$. The data were read out in a $2 \times 2$ pixel-binned fashion, in high gain mode.

The data were extracted and calibrated using the standard UVES data reduction pipeline, based on ECHELLE routines, which is part of the reduction package MIDAS (Ballester et al. 2000). This calibration process can introduce quasi-periodic oscillations in amplitude in the final spectral product (see Arav et al. 2008, on their analysis of QSO 2359-1241), which can create up to $2 \%-3 \%$ systematic errors in continuum placement. The observations were corrected to heliocentric and subject to a cosmic-ray removal procedure $(\sigma>3.5)$. We re-binned the data to $7.0 \mathrm{~km} \mathrm{~s}^{-1}$, approximately one resolution element, and normalized the spectrum using the methods described in Arav et al. (2008).

\section{DETECTION AND MEASUREMENTS}

The spectrum of SDSS J0318-0600 contains a plethora of absorption features available for potential diagnostics. In Figure 1, we show a sample of the VLT spectrum with line identifications and present the full spectrum with identifications in our online figure. These identifications come from matching the $\mathrm{Mg}$ II doublet lines and plotting location identifiers for other ionic lines. We also provide an online table for all identified lines, their wavelengths, oscillator strengths, and references; see Table 2. We show in Figure 1 a portion of the full online figure that contains readily visible lines from both the ground and excited levels of Fe II (lower energy levels up to $1873 \mathrm{~cm}^{-1}$ ).

\subsection{Identification of Kinematic Components}

We identify each of the individual kinematic absorption components in the spectrum using the Al II $\lambda 1671$ absorption line profile, which is a singlet transition with no nearby strong absorption features. In Figure 2, we show that there are approximately 11 separate kinematic components (labeled $\mathbf{a}-\mathbf{k}$ ), with component e being clearly a blend of multiple inseparable components. Using $\mathrm{Al}$ II as a template for the absorbers we search other ionic species for each of the 11 components.

Next, we identify the broad, strong $\mathbf{i}$ component in as many lines as possible and select unblended troughs from which we are able to measure ionic column densities. Figure 1 shows the UV 38 Fe II multiplet, which exhibits several absorption features from component $i$. We note that the absorption feature at $\sim 4727 \AA$ is the $\mathbf{k}$ component of the Fe II $\lambda 1608$ resonance line. While this identification scheme is sufficient to locate the majority of the lines that show an i component, we also use the Al II as a template (see Section 3.3) to search for the other 


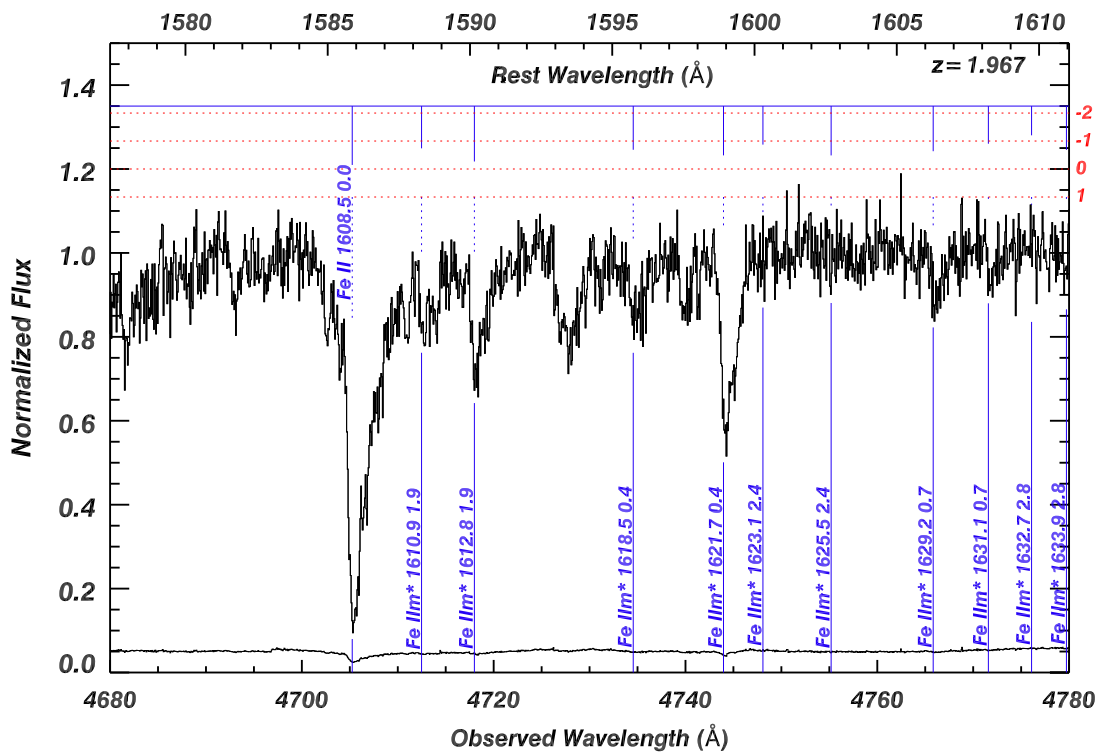

Figure 1. Portion of the VLT spectrum showing the Fe II UV 38 multiplet in SDSS J0318-0600 (the full spectrum can be found online). Line identifications are indicated by vertical blue lines for the expected position of component $\mathbf{i}$, based on the redshift $z=1.9257$. The redshift of the quasar is in black located at the top right. The $\log (g f)$ values of the identified transitions are shown above the spectrum, where $g$ is the statistical weight and $f$ is the oscillator strength. The size of the vertical blue line provides the value, which can be read from the $y$-axis scale at the top right. Each identifier lists the ion, wavelength, and lower energy level (in 1000 $\mathrm{s}$ of $\left.\mathrm{cm}^{-1}\right)$. The spectrum error is plotted in solid black below the spectrum $(\approx 0.05$ in normalized flux $)$

(A color version and the complete figure set [35 images] are available in the online journal.)

Table 2

Detected Absorption Lines in QSO 0318-0600

\begin{tabular}{lcccccc}
\hline \hline Ion & Wavelength & $\log g f$ & E $_{\text {low }}$ & glow $_{\text {low }}$ & $\begin{array}{c}\text { Components } \\
\text { Measured }\end{array}$ & Reference $^{\text {a }}$ \\
\hline C II & 1334.53 & -0.619 & 0 & 2 & $\mathbf{a}, \mathbf{b}-\mathbf{h}, \mathbf{k}$ & 5 \\
C II* & 1335.71 & -0.359 & 63 & 4 & $\mathbf{a}, \mathbf{i}, \mathbf{k}$ & 5 \\
C IV & 1548.20 & -0.423 & 0 & 2 & $\mathbf{a}, \mathbf{b}-\mathbf{h}, \mathbf{k}$ & 5 \\
C IV & 1550.77 & -0.723 & 0 & 2 & $\mathbf{i}, \mathbf{k}$ & 5 \\
\hline
\end{tabular}

Notes.

a (5) Moore (1970, Section 3).

(This table is available in its entirety in a machine-readable form in the online journal. A portion is shown here for guidance regarding its form and content.)

10 components in the Fe II UV 1, 2, \& 3 multiplet absorption complexes.

The Fe II multiplets UV 1, 2, \& 3 are the best candidates to identify Fe II lines from the other 10 components to measure, because they contain the $\mathrm{Fe}$ II transitions with the strongest oscillator strengths. However, this task proves to be nontrivial in SDSS J0318-0600. Identifying measurable lines from a saturated and heavily blended region (see Figure 2) is difficult, especially when considering the large number of strong resonance and excited state lines. Therefore, we attempt to identify as many of the 11 components in the UV 1, 2, and 3 multiplets using the $\mathrm{Al}$ II template to search for these 11 components where blending is not so prevalent. We provide the full list of detected ions, transition wavelengths, their oscillator strengths, and lower level energies in the online version of Table 2.

We find that for the lowest and highest velocity components (k and a), enough measurements or limit determinations are available and that we can effectively determine the electron density $\left(n_{H}\right)$, the ionization parameter $\left(U_{H}\right)$, and the total hydrogen column density $\left(N_{H}\right.$, see Section 4$)$. Unfortunately, blending prevents us from identifying enough measurable troughs for components $\mathbf{b}-\mathbf{h}$ for proper analysis. We opt for the limiting case by taking the sum of these latter systems (see Section 3.2).

\subsection{Column Density Determinations}

With a list of measurable absorption troughs, we extract ionic column densities. We begin the process by measuring lines from the strongest component, (i), where we employ three primary methods for determining the column densities. We describe three additional methods for column density determination that are less reliable in Section 3.3. We provide the measured values of ionic column densities as well as the method applied in Table 3.

Our first method of measuring column densities is to model a set of observed Fe II resonance lines (e.g., Figure 3) using an inhomogeneous absorption model, where we approximate the gas distribution across our line of sight (LOS) to the background source with a power law (see de Kool et al. 2002c; Arav et al. 2008, especially Figure 4, for a full description). The optical depth at a given velocity within the outflow is described by

$$
\tau_{v}(x)=\tau_{\max }(v) x^{a},
$$

where $x$ is the spatial dimension in the plane of the sky (simplified to one dimension without loss of generality, see Arav et al. 2005), $a$ is the power law distribution index, and $\tau_{\max }(v)$ is the highest value of $\tau$ at a given velocity. We simultaneously solve for both $\tau_{\max }$ and $a$ for each velocity resolution element of the resonance $\mathrm{Fe}$ II lines and apply this distribution $(a)$ to the excited Fe II lines. We then apply the same distribution dictated by the Fe II lines to all ions because this is the simplest assumption that imposes the strongest constraints.

We convert the modeled $\tau_{v}$ to column density via (Savage \& Sembach 1991)

$$
N(v)=3.8 \times 10^{14} \frac{1}{f \lambda} \tau_{v}\left(\mathrm{~cm}^{-2} \mathrm{~km}^{-1} \mathrm{~s}\right),
$$

where $\lambda$ is the wavelength of the line in $\AA, f$ is the oscillator strength, and $\tau_{v}$ is the optical depth in velocity space in $\mathrm{km} \mathrm{s}^{-1}$, which is integrated over the spatial dimension $x$. Using Equation (2), we compute the column density for each velocity element and integrate over the range in velocity, which we 

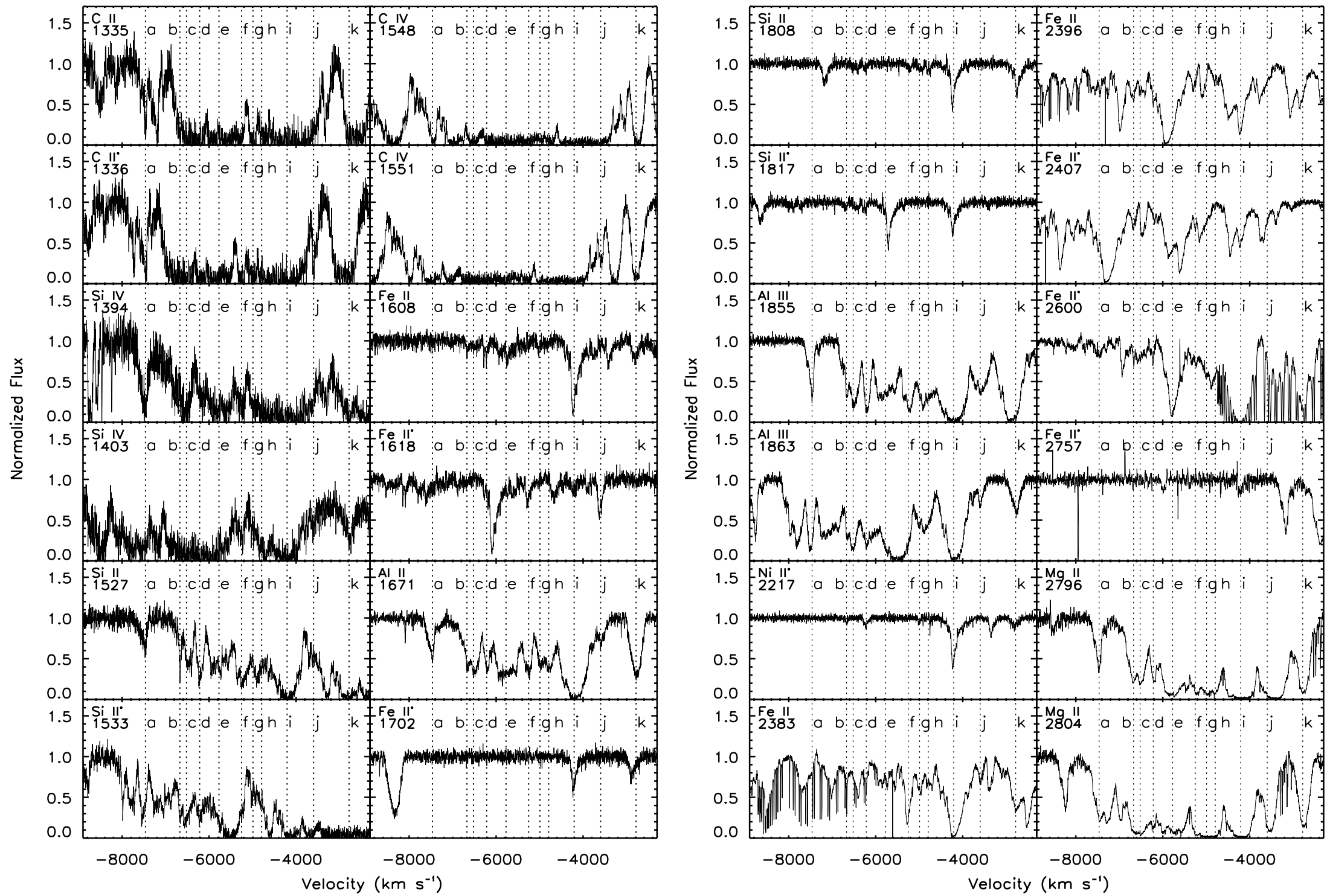

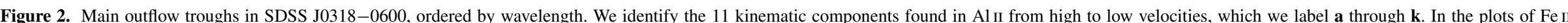

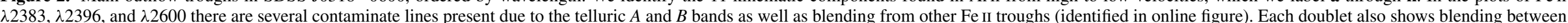
components (e.g., C IV, Mg II). 
Table 3

Component i: Measured Column Densities

\begin{tabular}{|c|c|c|c|c|c|c|}
\hline Ion & $\begin{array}{c}\text { Energy Level } \\
\left(E_{\text {low }} \mathrm{cm}^{-2}\right)\end{array}$ & $\begin{array}{c}\text { Velocity Range } \\
\left(\mathrm{km} \mathrm{s}^{-1}\right)\end{array}$ & $\begin{array}{l}\text { Column Density } \\
\left(\times 10^{12} \mathrm{~cm}^{-2}\right)\end{array}$ & $\begin{array}{l}\text { Uncertainty }^{\mathrm{a}} \\
\left(\times 10^{12} \mathrm{~cm}^{-2}\right) \\
\end{array}$ & $\begin{array}{l}\text { Number } \\
\text { of Lines }\end{array}$ & Methods ${ }^{b}$ \\
\hline$\overline{\mathrm{He}_{\mathrm{I}}}$ & 159856 & $-4600-3716$ & $<90$ & $\ldots$ & 1 & 6 \\
\hline $\mathrm{C}_{\mathrm{I}}$ & 0 & $-4600-3716$ & $<37$ & $\ldots$ & 1 & 6 \\
\hline $\mathrm{C}_{\text {II }}$ & 63 & $-4600-3714$ & $>19000$ & $\ldots$ & 1 & 4 \\
\hline C IV & 0 & $-4600-3714$ & 29000 & 3000 & 1 & 4 \\
\hline O I & 0 & $-4551-3896$ & 1900 & $\begin{array}{l}+2000 \\
-1200\end{array}$ & 1 & 5 \\
\hline $\mathrm{Mg}_{\mathrm{I}}$ & 0 & $-4600-3716$ & $<2$ & $\ldots$ & 1 & 6 \\
\hline Mg II & 0 & $-4600-3714$ & 3200 & 400 & 1 & 4 \\
\hline $\mathrm{Al}$ II & 0 & $-4477-3924$ & 400 & 40 & 1 & 2 \\
\hline Al III & 0 & $-4610-3833$ & 1560 & 220 & 1 & 3 \\
\hline Si II & 0 & $-4295-4106$ & 7220 & 720 & 1 & 1 \\
\hline Si II & 287 & $-4295-4106$ & 7380 & 740 & 1 & 1 \\
\hline Si IV & 0 & $-4600-3714$ & 5600 & 1300 & 1 & 4 \\
\hline Cr II & 0 & $-4295-4106$ & 14.6 & 1.5 & 2 & 1 \\
\hline Mn II & 0 & $-4295-4106$ & 17.5 & 1.8 & 1 & 1 \\
\hline $\mathrm{Fe}_{\mathrm{I}}$ & 0 & $-4600-3714$ & $<3$ & $\ldots$ & 1 & 6 \\
\hline Fe II & 0 & $-4295-4106$ & 1275 & 128 & 3 & 1 \\
\hline Fe II & 385 & $-4295-4106$ & 294 & 77 & 3 & 1 \\
\hline Fe II & 668 & $-4295-4106$ & $64^{\mathrm{d}}$ & $\ldots$ & 2 & 1 \\
\hline Fe II & 863 & $-4295-4106$ & $147^{\mathrm{d}}$ & $\ldots$ & 2 & 1 \\
\hline Fe II & 977 & $-4295-4106$ & $31^{\mathrm{d}}$ & $\ldots$ & 1 & 1 \\
\hline Fe II & 1873 & $-4295-4106$ & 163 & 16 & 4 & 1 \\
\hline Fe II & 2430 & $-4295-4106$ & 25 & 5.4 & 1 & 1 \\
\hline Fe II & 7955 & $-4295-4106$ & 8.1 & 0.8 & 3 & 1 \\
\hline Co II & 3351 & $-4295-4106$ & 9.4 & 0.9 & 1 & 1 \\
\hline Ni II & 0 & $-4295-4106$ & 180 & 18 & 3 & 1 \\
\hline Ni II & 8394 & $-4295-4106$ & 64 & 6.4 & 3 & 1 \\
\hline Zn II & 0 & $-4295-4106$ & 8.4 & 0.8 & 2 & 1 \\
\hline
\end{tabular}

Notes.

${ }^{a}$ Reflects the statistical errors from line fitting. This does not reflect the reliability of the measurement methods, which have increasing systematic error in order of increasing label number (see Section 3.2).

b Method used to determine column density, described by the respective label number: (1) power law fitting, (2) velocitydependent covering factor fitting, (3) apparent optical depth fitting, (4) "wing" fitting, (5) "tip" fitting, (6) upper limit based on the noise method; see Sections 3.2 and 3.3 for full details.

${ }^{c}$ Number of lines used in fitting. See online table for specific transition information.

d Unreliable measurements due to weak lines.

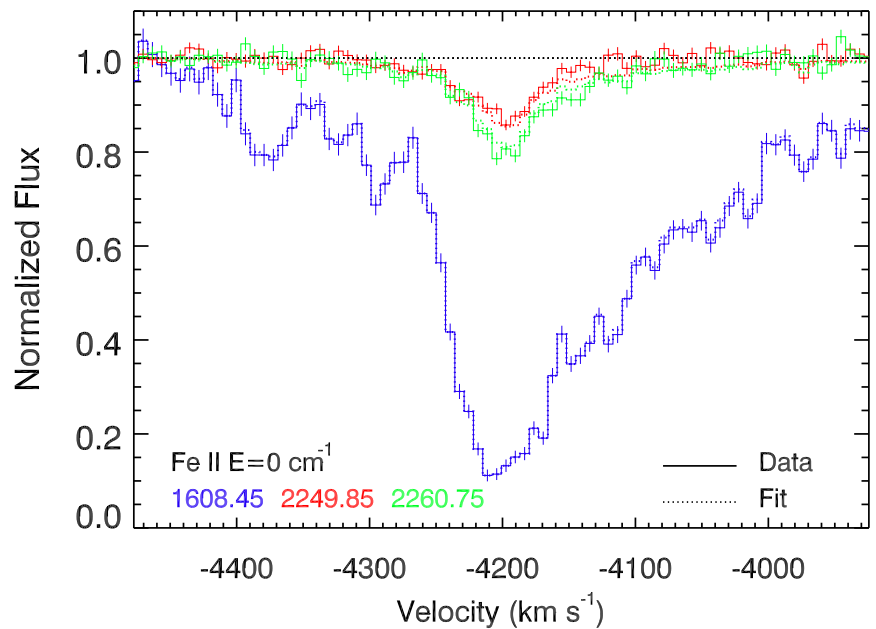

Figure 3. Line fits for the three uncontaminated Fe II resonance lines based on the power law method. The data are presented in $7 \mathrm{~km} \mathrm{~s}^{-1}$ bins with a solid line (statistical observational error bars for these data are also shown), while the model fit is shown by a dotted line. Each line is color coordinated with the wavelength located at the lower left of the plot.

justify later in this section. In Table 3, we quote the power law estimation of the column densities (when available) as this is the most accurate and most plausibly physical explanation of non- black saturated absorption at large distances (Arav et al. 2008; de Kool et al. 2002c). In Table 3 and throughout this paper, we will refer to this as method 1.

Method 2 is to model the Fe II resonance lines with a velocitydependent LOS covering factor. The LOS covering factor in this case is a geometric step-function distribution (see Figure 4 in Arav et al. 2008). In other words, a fraction of the extended source is completely obscured by the outflow, while the rest of the source is uncovered. We assume that each radial velocity element has an independent LOS covering factor, which we again determine using the Fe II resonance lines. We solve a fitting equation for $\tau_{v}$ (Arav et al. 2005),

$$
I(v)=1-C(v)+C(v) e^{-\tau(v)},
$$

where $I$ is the normalized intensity within the trough and $C(v)$ is the velocity-dependent LOS covering factor. First, we solve Equation (3) for $C(v)$ and $\tau(v)$ using at least two resonance lines from Fe II. We then calculate the velocity-dependent column density using $\tau(v)$ in Equation (2) and multiply the column density at each velocity by $C(v)$ to obtain the average column density across the LOS. Finally, we compute the total ionic column density by integrating across the velocity range of the trough. This method is comparable in accuracy to method 1 (Arav et al. 2008), and the column density results are approximately the same as found by method 1 . 


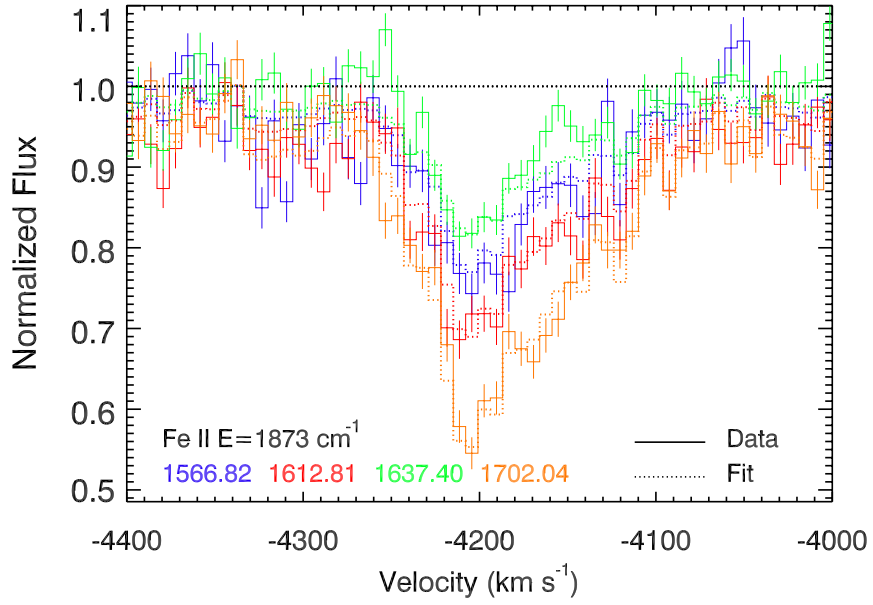

Figure 4. Power law model fits for four lines of metastable Fe II $\left(E_{\mathrm{low}}=\right.$ $1873 \mathrm{~cm}^{-1}$ ), we use the exponential $a$ parameter calculated from resonance Fe II. Similar to Figure 3.

The last of the three methods, method 3, is simply the apparent optical depth method (AOD; Savage \& Sembach 1991). This method assumes that all photons traveling to the observer pass through the same amount of gas at a given velocity, which gives the equation for normalized intensity: $I(v)=e^{-\tau(v)}$. We again convert to column density via Equation (2) and integrate to find the total ionic column density. This method does not account for partial LOS covering and saturation effects in line centers, which can underestimate column densities by up to an order of magnitude. However, we find that for SDSS J0318-066, in most cases a LOS covering factor of 1 (i.e., the AOD approximation) yields similar $N_{\text {ion }}$ values as those derived by the power law or partial covering method, a point we will discuss later in this section.

For the upcoming photoionization modeling, we adopt the ionic column densities as determined by method 1 (power law method) with some exceptions to this due to line blending that are noted in Table 3. We now present our analysis for the individual components.

\subsubsection{Measurements of Component $\boldsymbol{i}$}

We begin our column density determination by fitting the Fe II resonance lines from the strongest component, (i). In Figure 3, we show the fits for three $\mathrm{Fe}$ II resonance lines using the power law method. We select these particular line transitions because they provide us with a range of oscillator strengths and are free of blending or telluric contamination. Using these lines, we derive velocity-dependent column densities that we integrate for the total ionic column density.

Unfortunately, all strong Fe II lines from the lower energy level $385 \mathrm{~cm}^{-1}$ are heavily blended in the UV 1, 2, or 3 multiplets with lines from both the resonance and other excited lines (e.g., Fe II* $\lambda 2396$, shown in Figure 2), which renders column density measurements based on these transitions to have large systematic errors. However, we find relatively strong lines outside of the UV 1,2, or 3 multiplets for the $385 \mathrm{~cm}^{-1}$ energy state that are useful such as the Fe II line $\lambda 1622$ from the UV 38 multiplet. We also find two weaker lines from this energy state at $\lambda 1619$ and $\lambda 2281$ that are unblended. The oscillator strength values for these three lines are taken from Fuhr \& Wiese (2006). Of the three lines, both the $\lambda 1618$ and $\lambda 1622$ are reported to have accurate oscillator strengths (rated as " $\mathrm{A}$ " 8 by

\footnotetext{
8 Accuracies of "A," "B," and "C" are applied to oscillator strengths with
}

Fuhr \& Wiese), while the weaker $\lambda 2281$ has a reasonably reliable oscillator strength (rated "B"). However, we find that using the oscillator strengths reported by Fuhr \& Wiese is problematic. While the two weaker lines are well fit with these oscillator strengths, the trough depths relative to $\lambda 1622$ are unphysical. For example, scaling the $\lambda 1619$ trough in apparent optical depth by the ratio of oscillator strengths $(\sim 1.8)$ should be equal to the apparent optical depth in $\lambda 1622$ (or larger in the case of partial covering); however, the scaled optical depth of $\lambda 1619$ is significantly smaller than that of the $\lambda 1622$ trough. This means that at least one of the oscillator strengths could be less reliable than previously believed. Because of this issue, we quote an ionic column density for the $385 \mathrm{~cm}^{-1}$ energy level that is the mean of the fit to the $\lambda 1622$ line alone and the fit to the two weaker lines with an uncertainty equal to the range in column density.

The other excited state lines from Fe II (e.g., arising from 668,863 , and $977 \mathrm{~cm}^{-1}$ ) are also subject to heavy blending in the multiplets, but unlike the resonance and 385 excited state lines there is not sufficient column density to provide deep troughs with high signal-to-noise ratio for the weaker lines. Thus, the column density measurements from these energy levels are significantly more uncertain. The exceptions to this are lines from the lower energy level states $1873 \mathrm{~cm}^{-1}\left({ }^{4} F\right)$ and $7955 \mathrm{~cm}^{-1}\left({ }^{4} D\right)$.

We find five separate absorption troughs of Fe II arising from the level at $1873 \mathrm{~cm}^{-1}$, which are shown in Figure 4. We successfully fit these using the oscillator strengths from Kurucz \& Bell (1995) to determine the column density for this level. However, the compilation of recommended oscillator strengths of Fe II from Fuhr \& Wiese (2006) yields values lower than $\sim 0.3$ dex for two of the transitions, $\lambda 1637.4$ and $\lambda 1702.0$. For these transitions the recommended values are rated "C," while no oscillator strengths are given for the other two observed transitions. In addition to the difference in the absolute values, there is also a difference in the relative values, and in fact our fits to the absorption troughs in the spectrum favor the relative values of Kurucz \& Bell (1995). Thus, we opt to use the relative oscillator strengths from Kurucz \& Bell (1995) to fit all four troughs, but the level column density derived this way is increased by 0.3 dex, as required by the absolute oscillator strengths of Fuhr \& Wiese (2006).

We find that the results from all three models (Power law, covering factor, and AOD) are similar for the Fe II column density measurements of component $\mathbf{i}$, which suggests that the LOS covering factor of the system is close to 1 . Further evidence for a LOS covering factor $\approx 1$ case is shown in Figure 5 , where we show that we can use the $\mathrm{Al}$ II template to fit the $\mathrm{Al}$ III doublet. We scale two $\mathrm{Al}$ II templates (one for $\mathrm{Al}$ III $\lambda 1855$ and one for $\mathrm{Al}$ III $\lambda 1863$ ) in $\tau$ space for each individual kinematic component for the blue and the red line of the doublet by the expected ratio of $2: 1$ and coadd the templates to generate a fit to the data. There exist only small deviations, and the overall fit to the doublet is good. The most obvious and important deviation is in the fit to the $\lambda 1863 \mathbf{i}$ component, which is due to some saturation in the core of the Al II template. This demonstrates that AOD is an adequate approximation and the LOS covering factor for all components is approximately 1.0. This same procedure gives similar results when applied to the $\mathrm{Mg}$ II doublet as well.

uncertainties less than $3 \%, 10 \%$, and $25 \%$, respectively, according to the NIST Atomic Spectra Database. 


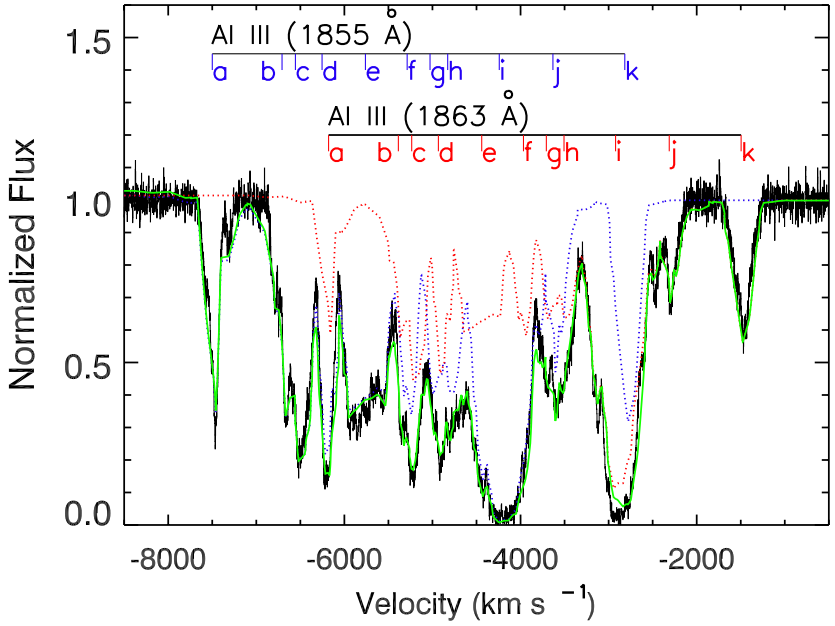

Figure 5. Al III doublet plotted in velocity space with respect to the blue member of the doublet. The blue and red tickmarks and letters indicate the component positions of the Al III doublet members, $\mathrm{Al}$ III $\lambda \lambda 1855,1863$ respectively. The dotted blue and red lines are the individual $\mathrm{Al}$ II template fits for each member of the doublet (corresponding to the blue and red tickmarks). Each individual component is scaled in $\tau$, and the resulting templates match the expected doublet ratio of 2:1 for each of the components. We coadd, in $\tau$ space, the two Al II templates and present the resulting fit in green. The fit agrees with the data with the exception of the $\lambda 1863$ component $\mathbf{i}$, which is likely due to saturation in the $\lambda 1855$ counterpart.

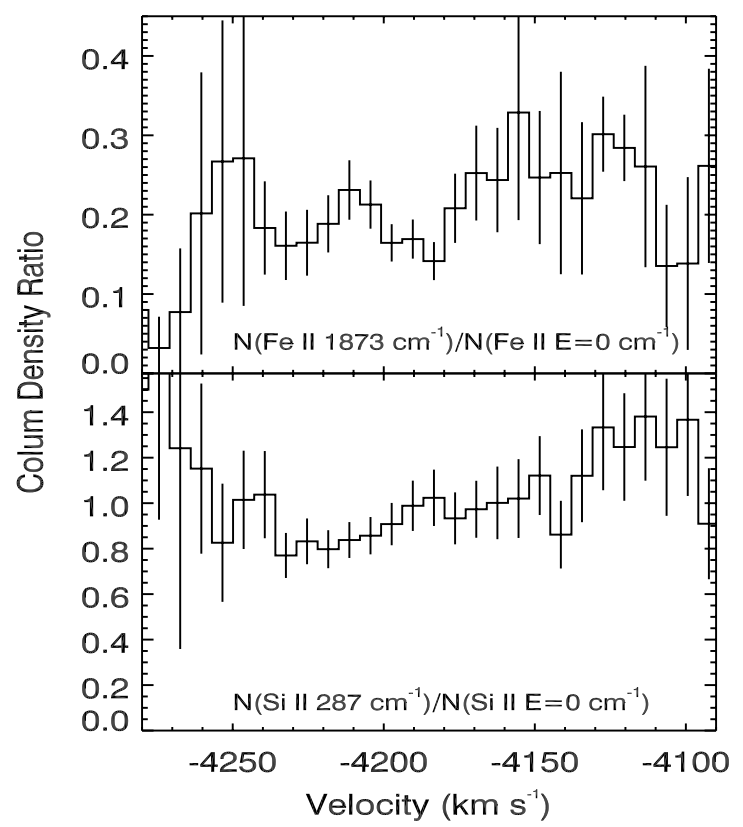

Figure 6. Component $\mathbf{i}$ column density ratios of $\mathrm{Fe}$ II*/Fe II and $\mathrm{Si}$ II*/Si II, top and bottom respectively, in velocity space. Within the error bars the ratios are constant across the integration range. This indicates that the outflowing gas has a constant electron number density across the trough, which strongly suggests similar ionization conditions across the velocity span of this outflow component.

\subsubsection{The Physical Connection Between Material Seen in Different Troughs}

Ultimately, we want to establish the physical characteristics of the outflow based on the column density determinations described above. In order to do so, we use the integrated column densities as input to photoionization models, which in turn determine the total hydrogen column density and number density in the outflow. This procedure depends on two assumptions: (1) that the physical conditions do not change as a function of velocity across a given trough. This assumption allow for the use of integrated column densities instead of

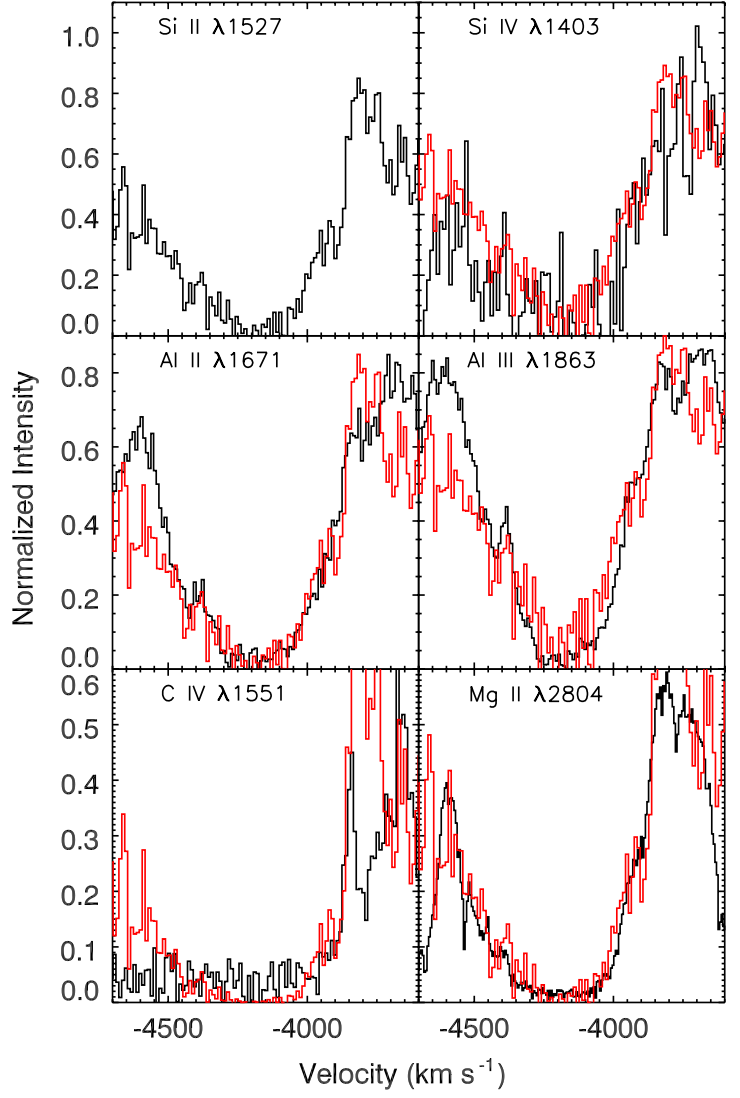

Figure 7. Top left panel shows the Si II $\lambda 1527 \mathbf{i}$ component. The remaining panels show the agreement of the Si II $\lambda 1527 \mathbf{i}$ component trough with the red doublet member of five other ions. The Si II $\lambda 1527$ data have been converted to $\tau(v)$, where $\tau(v)=-\ln (I(v))$, and scaled by a single factor for each of the other lines in order to produce the fits (red histograms) for these troughs (data shown in black). Some discrepancy between the Si II $\lambda 1527$ line and Al III $\lambda 1863$ occurs near $4000 \mathrm{~km} \mathrm{~s}^{-1}$, which is due to contamination from the blue $\mathbf{k}$ component. These agreements show that the ratio of column densities between each ion and $\mathrm{Si}$ II is constant across the profiles of the different troughs.

working with each velocity resolution element separately (see Arav et al. 2008); (2) that the troughs of different ions correspond to the same outflowing gas, which is a necessary condition for using these measurements in the same photoionization models. Both assumptions can be checked using the data at hand.

The first assumption can be tested by checking whether the ratio of measured column densities among different lower levels of the same ion vary as a function of velocity. In Figure 6, we plot the ratios of column densities between those arising from excited levels to those from the ground state level of Fe II and $\mathrm{Si}$ II. Within the uncertainties, the ratios across the troughs are velocity independent, showing that the electron number density is constant across the flow (see Section 4 for elaboration). This result gives validity to our first assumption and justifies the use of integrated column densities for component $i$. We also tested this for $\mathrm{Ni}$ II/Ni II* and combinations between Ni II, Si II, and Fe II resonances and excited lines and find that the ratio is constant in all cases. This constant ratio is also seen in the results for the outflow system in QSO 2359-1241 as described by Korista et al. (2008).

In order to validate the second assumption, we need to check the column density ratio across troughs from different ions and especially to compare low- and high-ionization troughs. To do so, we use the $\tau(v)$ profile of $\mathrm{Si}$ II $\lambda 1527$ and attempt to fit the profiles of other troughs while using a single-scale factor for each line. The results are shown in Figure 7. It is evident that the 


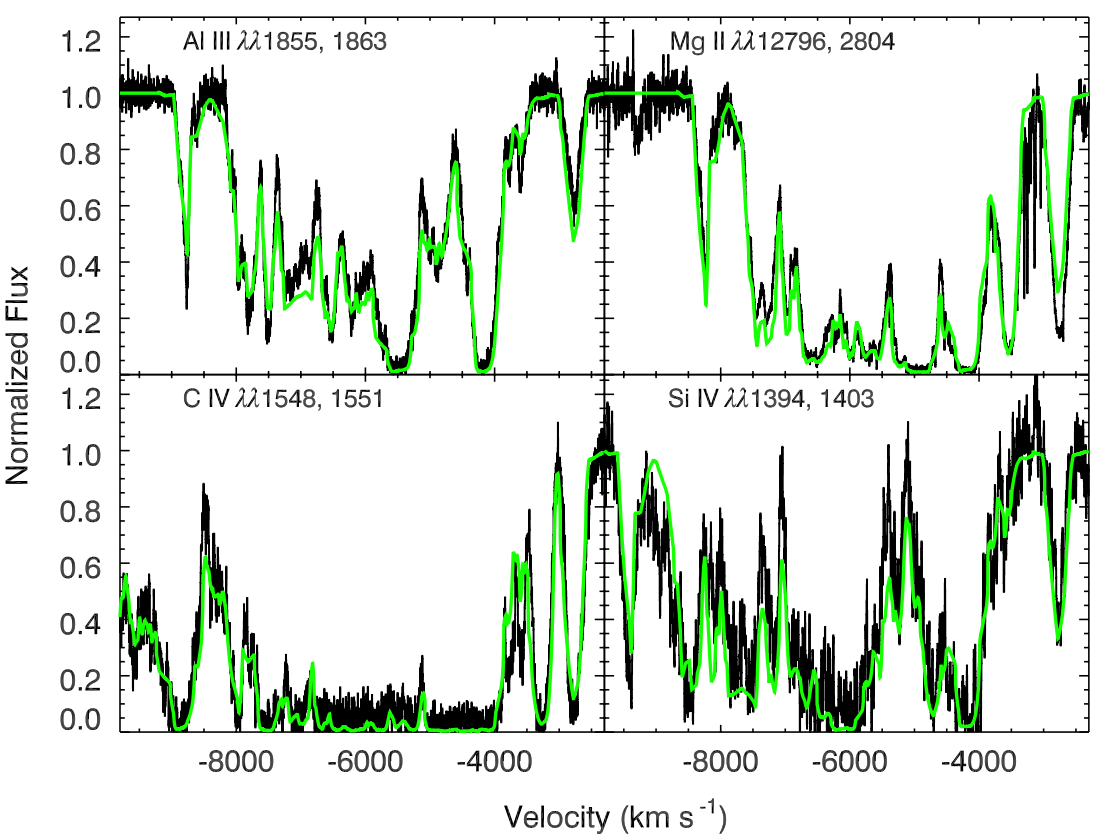

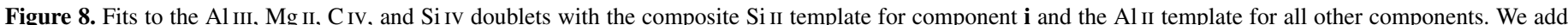

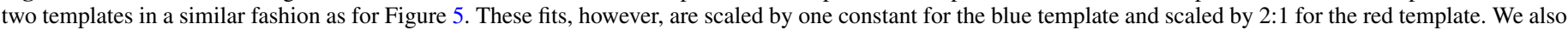

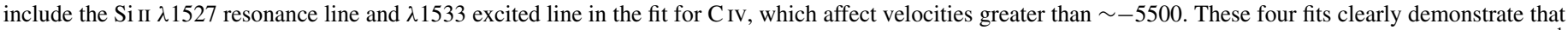

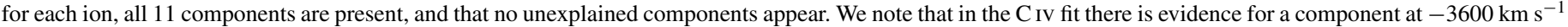

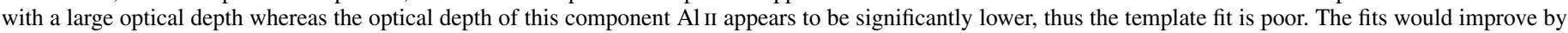
scaling each component by a different factor in optical depth.

scaled $\tau(v)$ profile of Si II $\lambda 1527$ fits the troughs of Si IV, Al II, $\mathrm{Al}$ III troughs and $\mathrm{Mg}$ II remarkably well, lending strong support for the assumption that we are measuring the same outflowing gas. For C IV, which is the highest ionization line observed, the fit for the center of the trough is less conclusive since the trough is highly saturated at its core. However, we point out that the fit to the low-velocity wing $\left(-3950\right.$ to $\left.-3850 \mathrm{~km} \mathrm{~s}^{-1}\right)$ is very good, which would be a coincident unless the C IV trough can be fitted with the same $\tau(v)$ profile of Si II $\lambda 1527$. In summary, the data support the two main assumptions that we need in order to use all integrated column densities in our photoionization models.

To demonstrate the tie between the high- and low-ionization troughs for the other outflow components, we show in Figure 8 that the combination of the Si II composite template (described in Section 3.3 for component i) and the Al II template (for all other components) can be used to recreate the doublet troughs of Mg II, Al III, Si IV, and C IV. Similar to Figure 5, we add two templates together in $\tau(v)$. We scale the entire blue template (all 11 components) by one factor and scale the respective red template by 2:1 in $\tau(v)$. This method gives the fewest possible constraints for the fit. We find that all 11 kinematic components are present in both low- and high-ionization troughs and that there are no unexplained kinematic components in the higher ionization state lines, which is indication that this is the same outflowing gas for both the high- and low-ionization troughs. We also note that these fits could be better if we scaled each individual component for the blue member and kept the 2:1 ratio to the red, as we did for the $\mathrm{Al}$ III fit in Figure 5.

\subsubsection{Measurements of the Other Components}

The other two components with a sufficient number of measurable absorption lines are the $\mathbf{k}$ and $\mathbf{a}$ components. However, we are unable to utilize methods 1 or 2 due to a lack of unblended Fe II resonance lines. For component $\mathbf{k}$, only one $\mathrm{Fe}$ II resonance line (Fe II 21608 ) is measurable and unblended. Thus, we measure the column densities based on a LOS covering factor of 1 . We further confirm this assumption of a LOS covering factor of 1 , as with the $\mathbf{i}$ component, by checking that the $\lambda 1608$ line scales well to the unblended portion of Fe II $\lambda 2383$ according to oscillator strength. Component a has no measurable Fe II resonance lines. However, according to the $\mathrm{Al}$ III doublet (Figure 5), we find that a LOS covering of $\sim 1$ holds for all components and we are able to resort to the AOD method for our measurements. The results for components $\mathbf{k}$ and $\mathbf{a}$ are shown in Tables 4 and 5, respectively.

Due to the lack of measurable, unblended troughs for components $\mathbf{b}$ through $\mathbf{h}$, we use the apparent optical depth method and integrate the total column density across the combined blended troughs for components b through $\mathbf{h},-7150$ to $-4600 \mathrm{~km} \mathrm{~s}^{-1}$ in velocity, for the $\mathrm{Si}$ IV, $\mathrm{C}_{\text {IV }}, \mathrm{Mg}_{\mathrm{II}}$, and $\mathrm{Al}$ III doublets as well as the resonance and excited lines of $\mathrm{C}_{\mathrm{II}}(\lambda 1335, \lambda 1336)$ and Si II $(\lambda 1527, \lambda 1533)$. We present these results in Table 6.

\subsection{Estimates and Limits}

For situations where blending between components in doublets is prevalent (i.e., $\mathrm{C}_{\mathrm{IV}}, \mathrm{Si}$ IV, $\mathrm{C}_{\mathrm{II}}$, and $\mathrm{C}_{\mathrm{II}}{ }^{*}$ ) or where the trough is heavily saturated (i.e., C IV), our primary method of column density extraction fails. Thus, we resort to other, less accurate, methods to estimate the column densities, which are important when constraining photoionization models. We develop three additional methods in order to estimate column densities in blended regions, which we explain here in order of decreasing accuracy.

In the first of our estimation methods (4), we opt to employ a template to map out approximate profiles for the troughs. We utilize two templates for this process. The first template is a fit to the Al II $\lambda 1671$ line for all 11 components. However, due to moderate saturation in the core of the $\mathrm{Al}$ II $\mathbf{i}$ component, we create a composite template using the $\mathrm{Si}$ II resonance lines for the $\mathbf{i}$ component. 
Table 4

Component k: Measured Column Densities

\begin{tabular}{|c|c|c|c|c|c|c|}
\hline Element & $\begin{array}{c}\text { Energy Level } \\
\left(E_{\text {low }} \mathrm{cm}^{-2}\right)\end{array}$ & $\begin{array}{c}\text { Velocity Range } \\
\left(\mathrm{km} \mathrm{s}^{-1}\right)\end{array}$ & $\begin{array}{l}\text { Column Density } \\
\left(\times 10^{12} \mathrm{~cm}^{-2}\right)\end{array}$ & $\begin{array}{c}\text { Uncertainty } \\
\left(\times 10^{12} \mathrm{~cm}^{-2}\right)\end{array}$ & $\begin{array}{l}\text { Number } \\
\text { of Lines }^{\mathrm{a}}\end{array}$ & Methods ${ }^{b}$ \\
\hline $\mathrm{C}_{\text {II }}$ & 0 & $-3088-2249$ & 1100 & 200 & 1 & 4 \\
\hline $\mathrm{C}_{\mathrm{II}}$ & 63 & $-3088-2249$ & 1950 & 300 & 1 & 4 \\
\hline C IV & 0 & $-2893-2641$ & 1297 & 130 & 2 & 3 \\
\hline Mg II & 0 & $-2893-2641$ & 192 & 20 & 1 & 3 \\
\hline Al II & 0 & $-3047-2494$ & 35 & 4 & 1 & 3 \\
\hline Al III & 0 & $-3047-2494$ & 73 & 7 & 1 & 3 \\
\hline Si II & 0 & $-3321-2262$ & 640 & 150 & 1 & 6 \\
\hline Si II & 287 & $-2893-2641$ & 352 & 40 & 1 & 3 \\
\hline Si IV & 0 & $-2893-2641$ & 140 & 20 & 1 & 3 \\
\hline Fe II & 0 & $-2893-2641$ & 154 & 20 & 1 & 3 \\
\hline Fe II & 668 & $-2893-2641$ & 9.0 & 1.0 & 1 & 3 \\
\hline Ni II & 0 & $-3199-2502$ & $<120$ & $\ldots$ & 1 & 6 \\
\hline $\mathrm{Ni}$ II & 8394 & $-2893-2641$ & 10.0 & 1.0 & 1 & 3 \\
\hline
\end{tabular}

Notes.

a Number of lines used in fitting. See online table for specific transition information.

b Method used to determine column density, described by the respective label number: (1) power law fitting, (2) velocitydependent covering factor fitting, (3) apparent optical depth fitting, (4) "wing" fitting, (5) "tip" fitting, (6) upper limit based on the noise method.

Table 5

Component a: Measured Column Densities

\begin{tabular}{|c|c|c|c|c|c|c|}
\hline Element & $\begin{array}{c}\text { Energy Level } \\
\left(E_{\text {low }} \mathrm{cm}^{-2}\right)\end{array}$ & $\begin{array}{c}\text { Velocity Range } \\
\left(\mathrm{km} \mathrm{s}^{-1}\right)\end{array}$ & $\begin{array}{l}\text { Column Density } \\
\left(\times 10^{12} \mathrm{~cm}^{-2}\right)\end{array}$ & $\begin{array}{c}\text { Uncertainty } \\
\left(\times 10^{12} \mathrm{~cm}^{-2}\right)\end{array}$ & $\begin{array}{l}\text { Number } \\
\text { of Lines }\end{array}$ & Methods ${ }^{b}$ \\
\hline $\mathrm{C}_{\text {II }}$ & 0 & $-7523-7271$ & 333 & 30 & 1 & 3 \\
\hline $\mathrm{C}_{\text {II }}$ & 63 & $-7523-7271$ & 577 & 60 & 1 & 3 \\
\hline C IV & 0 & $-7558-7243$ & 734 & 70 & 1 & 3 \\
\hline Mg II & 0 & $-7523-7271$ & 28.7 & 2.9 & 1 & 3 \\
\hline $\mathrm{Al}$ II & 0 & $-7523-7271$ & 11.6 & 1.2 & 1 & 3 \\
\hline Al III & 0 & $-7523-7271$ & 46.0 & 5.0 & 1 & 3 \\
\hline Si II & 0 & $-7523-7271$ & 101 & 10 & 1 & 3 \\
\hline Si II & 287 & $-7523-7271$ & $<50^{\mathrm{c}}$ & $\cdots$ & 1 & 5 \\
\hline Si IV & 0 & $-7523-7271$ & 145 & 20 & 1 & 3 \\
\hline Fe II & 0 & $-7695-7300$ & $<40$ & $\ldots$ & 1 & 6 \\
\hline
\end{tabular}

Notes.

a Number of lines used in fitting. See online table for specific transition information.

b Method used to determine column density, described by the respective label number: (1) power law fitting, (2) velocitydependent covering factor fitting, (3) apparent optical depth fitting, (4) "wing" fitting, (5) "tip" fitting, (6) upper limit based on the noise method.

c Column limit based on the limiting ratio "tip" fit for Si II*/Si II.

Table 6

Components b-h: Column Densities Limits

\begin{tabular}{lcccc}
\hline \hline Element & $\begin{array}{c}\text { Energy Level } \\
\left(E_{\text {low }} \mathrm{cm}^{-2}\right)\end{array}$ & $\begin{array}{c}\text { Velocity Range } \\
\left(\mathrm{km} \mathrm{s}^{-1}\right)\end{array}$ & $\begin{array}{c}\text { Column Density } \\
\left(\times 10^{12} \mathrm{~cm}^{-2}\right)\end{array}$ & $\begin{array}{c}\text { Number } \\
\text { of Lines }\end{array}$ \\
\hline C II $_{\text {II }}$ & 0 & $-6950-4400$ & $>13000$ & 1 \\
C IV & 0 & $-7150-4600$ & $>9900$ & 1 \\
Mg II & 0 & $-7150-4600$ & $>910$ & 1 \\
Al II & 0 & $-7150-4600$ & $>440$ & 1 \\
Al III & 0 & $-7150-4600$ & $>840$ & 1 \\
Si II & 0 & $-7150-4600$ & $>3200$ & 1 \\
Si IV & 0 & $-7150-4600$ & $>1900$ & 1 \\
Fe II & 0 & $-7150-4600$ & $>600$ & 1 \\
\hline
\end{tabular}

Note. ${ }^{\text {a }}$ Number of lines used in fitting. See online table for specific transition information.

To create this template, we use both the Si II $\lambda 1808$ and $\lambda 1527$ lines. The $\lambda 1527$ line is similar in shape to the Al II $\lambda 1671$ line, and while the core is moderately saturated, it provides us with a good fit to the wings of the trough. In contrast, the $\lambda 1808$ line has a significantly weaker oscillator strength and therefore the core of this trough is not saturated; however, the wings are buried in the noise. We therefore create a composite template using the AOD column densities of the $\lambda 1527$ for the wings $\left(-3820\right.$ to $-3990 \mathrm{~km} \mathrm{~s}^{-1}$ and -4390 to $\left.-4580 \mathrm{~km} \mathrm{~s}^{-1}\right)$, and the AOD column densities of the $\lambda 1808$ line for the core $(-4390$ and $-3990 \mathrm{~km} \mathrm{~s}^{-1}$ ). We then convert the resultant Si II column density profile to $\tau(v)$ template for the $\lambda 1527$ line.

To measure column densities, we scale this composite $\tau(v)$ template to match the red wings of the $\mathrm{Mg}$ II, Si IV, and C IV doublets as well as $\mathrm{CII}^{*}$, which are apparently free of blending. We assume that the column density ratio is constant across the troughs, which is supported by Figure 7, scale the composite template for the $\mathbf{i}$ component to match and then integrate the total column (assuming a $C_{\mathrm{los}} \approx 1$ ) from the template. We refer to this method as the "wing" fitting method (method 4). The error estimation is provided by using two $\tau$ scaling values, one underestimating and one overestimating the noise level in the trough's wing. We note that these measurements use only one line, the red doublet member, which is reflected in Tables 3 through 6 . To further test the validity of this method, we used the composite template to fit the wing of the Si II $\lambda 1527$ line 
and we find that the template is able to reproduce the column density we find in our power law fitting of the $\lambda 1808$ line to within $10 \%$.

The next method we designate as the "tip" fitting method, or method 5 , where we estimate the column densities in a similar way to method 4 . For example, we estimate the column density of $\mathrm{O}$ I $\lambda 1302$ for the $\mathbf{i}$ component in the region containing blended lines from Si II $\lambda 1304$ and Si II* $\lambda 1309$. We find that the composite template fit for the $\mathbf{i}$ component agrees well with the deep trough at $\sim 3809 \AA$ in velocity space (shown in online Figure 1), which appears to be the tip of $\mathrm{O}$ I absorption. Thus, we use the composite Si II template to approximate the minimum and maximum amount of column density that could come from $\mathrm{O}$ I. Due to the uncertainty in continuum placement and possible saturation, we consider this method less accurate than method 4 , which is reflected in the larger error bars.

Our last column density estimation method (the noise method or method 6) defines upper limits on the column density of ions based on the statistical error of the data. Because the column density of $\mathrm{He} \mathrm{I}$ is important for photoionization models (see Arav et al. 2001; Korista et al. 2008), we estimate an upper limit for the column density of the excited $2^{3}$ s level of He I $\left(\mathrm{He} \mathrm{I}^{*}\right.$ hereafter) in component $\mathbf{i}$ for $\mathrm{He} \mathrm{I}^{*} \lambda 3189$. To do this, we scale the Si II template for the $\mathbf{i}$ component and compare it to the region where the line would be detected. We vary the scaling to the noise level, find the largest possible optical depth that could be buried without detection and integrate across the template to determine the column density. We also perform this analysis for $\mathrm{Mg}_{\mathrm{I}} \lambda 2853, \mathrm{Fe}_{\mathrm{I}} \lambda 2484$, and $\mathrm{C}_{\mathrm{I}} \lambda 1560$ and quote the measured limits in Table 3.

Component $\mathbf{k}$ presents its own problems. Unlike the $\mathbf{i}$ component, here we effectively measure column densities for Fe II resonance and excited levels $\left(E=0 \mathrm{~cm}^{-1}\right.$ to $\left.E=668 \mathrm{~cm}^{-1}\right)$, but we lack a Si II resonance line to provide a ratio to the $\mathrm{Si}$ II $\lambda 1309$ excited line. Therefore, we apply method 5 with the Al II template to find limits on the Si II. Moreover, we use the "wing" fitting method (method 4) with the same Al II template to estimate the column density for $\mathrm{C}$ II and $\mathrm{C}$ II* in component $\mathbf{k}$.

In component $\mathbf{a}$, the number of measurable lines is even fewer than for component $\mathbf{k}$. The only clean, unblended density diagnostic is $\mathrm{C}$ II to $\mathrm{CII}^{*}$. As the critical density for this ratio is low $\left(\sim 10^{2} \mathrm{~cm}^{-3}\right.$, Blum \& Pradhan 1992; Froese Fischer 1983), it is important to find another density check. Thus, using the Al II template, we estimate an upper limit to the column density ratio of Si II* to $\mathrm{Si}$ II to be 0.49 for component $\mathbf{a}$.

\subsection{Systematic Uncertainty}

We recognize that there exist systematic errors that could contribute further to the uncertainty in our measurements. For example, our placement of the continuum, defined in Section 2, could result in increased errors of the Si II $\lambda 1808$ and Si II* $\lambda 1817$ column densities by $5 \%$. The amount of saturation in the core of $\mathrm{Si}$ IV is another systematic error that could be a potential source of error in column density, which at maximum, could lead to an error in an ionization parameter of 0.1 dex. Both of these are negligible compared to the uncertainties due to the chemical abundance choice.

\section{SPECTRAL DIAGNOSTICS AND MODELING}

The observed spectrum of SDSS J0318-0600 exhibits distinct absorption from $\mathrm{C}_{\mathrm{II}}$ and $\mathrm{C}$ IV, $\mathrm{O}_{\mathrm{I}}, \mathrm{Mg}_{\mathrm{II}}, \mathrm{Al}$ II and $\mathrm{Al}$ III, Si II and Si IV, Cr II, Fe II, Co II, Ni II, and Zn II for component i.
In addition, we measure upper limits to the column densities of $\mathrm{He}^{*}$, Fe I, C I, and Mg I. As already shown, a similar absorption trough structure is shared by the strongest transitions of the singly ionized species, and those of intermediate ionization (Al III and Si IV), as well as C IV. Additionally, component i likely has the largest column density of any component in SDSS J0318-0600, given that it has the largest number of absorption line identifications and is the deepest absorption feature for transitions of low oscillator strengths and/or low relative elemental abundance. Therefore, we analyze component $\mathbf{i}$ here, and analysis of all other absorption components will be presented in a subsequent paper (M. Bautista et al. 2010, in preparation).

One of the main objectives of modeling the observed ionic column densities is the determination of the total hydrogen column density of the outflow $N_{H}$ and its distance from the central source $R$, which are key in the determination of kinetic luminosity of the outflow (see Section 6). The former is obtained directly through photoionization modeling, but the latter is not. Instead, modeling yields the so-called ionization parameter defined as

$$
U_{H}=\frac{Q_{H}}{4 \pi R^{2} n_{H} c}=\frac{\Phi_{H}}{n_{H} c},
$$

where $n_{H}$ is the total hydrogen density, $c$ is the speed of light, $\Phi_{H}$ is the surface flux of ionizing photons, $Q_{H}$ is the rate of hydrogen ionizing photons emitted by the central object, which is defined by

$$
Q_{H}=\int_{1 R y}^{\infty} \frac{L_{v}}{h v} d \nu\left(\text { ionizing photons s}{ }^{-1}\right),
$$

where the integration range covers the range of ionizing photons, $L_{v}$ is the luminosity at each frequency, and $h$ is the Planck constant. From this definition, $Q_{H}$ can be estimated directly from the observed luminosity ( $\lambda L_{\lambda}$ of the object and some knowledge of its spectral energy distribution (SED), see Sections 4.2 and 4.3). Thus, the distance $R$ can be obtained from the diagnosed value of $U_{H}$ whenever one has an independent determination of $n_{H}$, which in a cloud with fully ionized hydrogen is roughly equal to the electron density, $n_{e}$.

Thus, the first step in the analysis is to determine $n_{e}$ from its effect via collisions on the relative populations of excited levels observed in the spectrum. Later, we discuss the nature of the SED, which is to be used in the determination of $Q$ and as input for photoionization modeling. After this, we will proceed to detailed photoionization modeling.

\subsection{Electron Density Determination}

Under excitation equilibrium conditions, the column densities of lowly excited metastable levels can be used as diagnostics of electron density, assuming that the atomic parameters and excitation processes responsible for excitation are sufficiently well understood. We have measured column densities for the $0 \mathrm{~cm}^{-1}$ ground state and low-lying metastable excited levels within Si II, Fe II, and Ni II.

The simplest and probably most reliable of the electron density diagnostics is that from the ratio of the column densities of the Si II levels, as shown in Figure 9. Here, the ratio of the excited $3 \mathrm{~s}^{2} 3 \mathrm{p}^{2} \mathrm{P}_{3 / 2}^{o}$ at $287 \mathrm{~cm}^{-1}$ to the $3 \mathrm{~s}^{2} 3 \mathrm{p}{ }^{2} \mathrm{P}_{1 / 2}^{o}$ ground level is calculated from three different sets of effective collision strengths, Dufton \& Kingston (1991), Tayal (2008), and M. Bautista et al. (2010, in preparation). We measure this column density ratio from the pair of absorption lines at $1808.0 \AA$ (resonant) and $1816.9 \AA$ (excited state). As described above, 


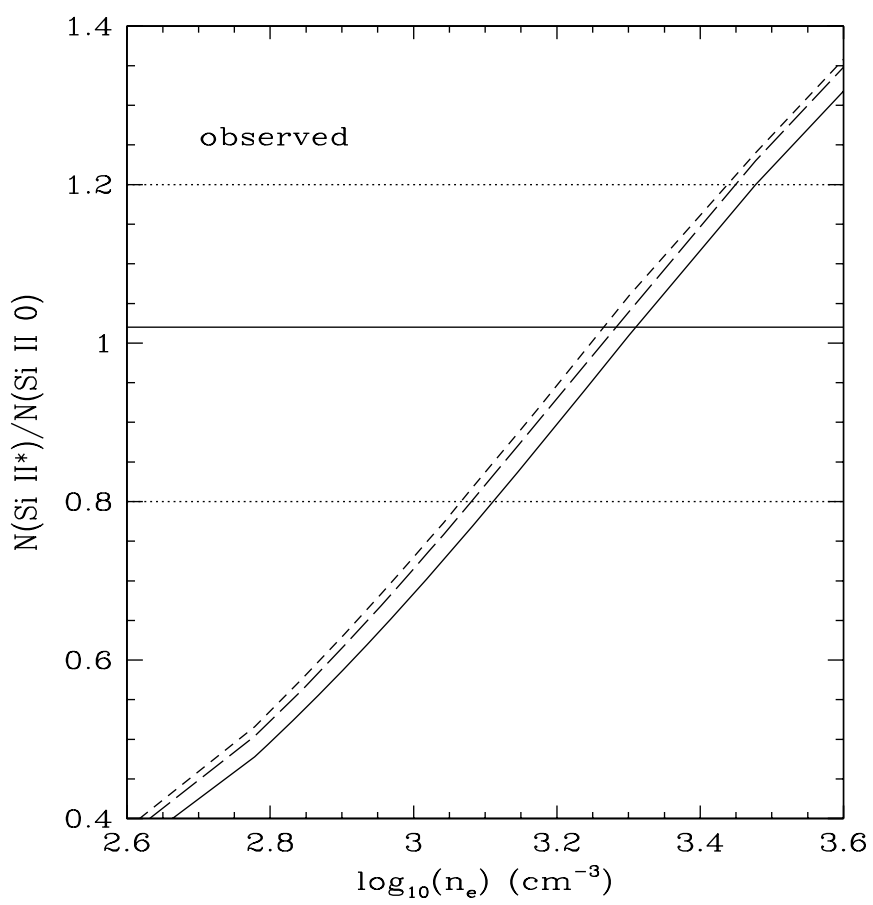

Figure 9. Electron density diagnostic ratio of the populations of the first excited level of Si II and the ground state. The theoretical line ratios are calculated at $T_{e}=10,000 \mathrm{~K}$, although the ratio is rather insensitive to this choice. The various curves depict the ratios obtained from the collision strengths of Dufton \& Kingston (1991, solid curve), Tayal (2008, long-dashed curve), and M. Bautista et al. (2010, in preparation, short-dashed curve). The horizontal lines indicate the observed ratio (heavy solid) and estimated uncertainties (dotted), which are dominated by the uncertainties in the oscillator strengths of the transition pair at $1808.0 \AA$ and $1816.9 \AA$.

we have a good deal of confidence in their measured integrated optical depths, especially their ratio, and we will thus place the greatest weight on this electron density diagnostic. We caution, however, that as the weakest among such pairs of transitions commonly measured in Si II, there are moderate uncertainties in their oscillator strengths, even in their relative values (about $0.1 \mathrm{dex}$ ). Two other such pairs of transitions that lie within our spectrum, centered on $1307 \AA$ and $1531 \AA$, have smaller uncertainties in their oscillator strengths but are saturated in our spectrum and are not useful for direct measurements. These uncertainties dominate over the statistical measurement errors in the column density fits to the observed troughs at $1808.0 \AA$ and 1816.9 Å. Figure 9 illustrates the electron density dependence of this level specific column density ratio, wherein the solid and dotted horizontal lines represent our measurement of this ratio and an estimated range of its uncertainty, the latter dominated by the uncertainties in the transition oscillator strengths. We derive an electron density of $\log \left(n_{e}\right)=3.3 \pm 0.2\left(\mathrm{~cm}^{-3}\right)$.

The various observed transitions arising from low-lying metastable levels of the Fe II ion allow for independent electron density diagnostics. However, constraining the electron density from the relative populations within these metastable levels proved more difficult. We present these in Figure 10 for column densities of the $385 \mathrm{~cm}^{-1}, 1873 \mathrm{~cm}^{-1}$, and $7955 \mathrm{~cm}^{-1}$ levels. These are the only levels for which we have sufficient confidence in their measured column densities (see Table 2) to justify their utility as electron density diagnostics. In blue are the predictions from CLOUDY (ver. 06.02; Ferland et al. 1998) for photoionization models presented in Korista et al. (2008) for the main outflow system in QSO J2359-1241 (solar abundances, fixed ionization parameter, and total column density for which no hydrogen ionization front formed, and $n_{e} \approx n_{H}$ within the $\mathrm{Fe}^{+}$zone). Relevant mainly for the ratios involving the $7955 \mathrm{~cm}^{-1}$ level, the electron density weighted average temperature within the $\mathrm{Fe}^{+}$zone of these models was approximately $11,000 \mathrm{~K}$. In red are the predictions of the collisional equilibrium model $\mathrm{Fe}$ II atom of Verner et al. (1999) for a fixed electron temperature $(10,000 \mathrm{~K})$ at each electron density. This difference in adopted temperature has no significant effect on the populations of the $385 \mathrm{~cm}^{-1}$ and $1873 \mathrm{~cm}^{-1}$ levels, while the population of the $7955 \mathrm{~cm}^{-1}$ level is roughly linearly dependent on electron temperature for the conditions of interest (e.g., $\sim 10 \%$ differences are found for the two temperatures quoted). The horizontal solid and dashed lines are the measured values and estimated uncertainties, respectively. The small differences in the predictions pertaining to the $385 \mathrm{~cm}^{-1}$ level and the large differences pertaining to the $1873 \mathrm{~cm}^{-1}$ level are primarily due to differences in the radiative transition rates ( $A$-values) for forbidden transitions used in the two sets of computations. A detailed inspection of the atomic data files of the Verner et al. (1999) model atom in CLOUDY revealed that for a few dipole forbidden transitions the model adopts $A$-values whose sources are neither known to us nor cited in their publication. For all other relevant transitions, the Verner et al. model atom in CLOUDY adopts the $A$-values from Quinet et al. (1996). ${ }^{9}$ The largest effects of the $A$-values of unknown origin present in CLOUDY are with respect to the population of the $1873 \mathrm{~cm}^{-1}$ level, where the differences are substantial as indicated in the middle panel of Figure 10 (blue and red curves). ${ }^{10}$

The electron density predicted by the Si II lines $\left(\log \left(n_{e}\right)=\right.$ $3.3 \pm 0.2 \mathrm{~cm}^{-3}$; see Figure 9) and that from the $385 \mathrm{~cm}^{-1}$ level in Fe II $\left(\log \left(n_{e}\right) \approx 3.9 \mathrm{~cm}^{-3}\right)$ differ somewhat in value. However, considering the moderate uncertainties in the relevant atomic data of the $\mathrm{Si}$ II and $\mathrm{Fe}$ II $385 \mathrm{~cm}^{-1}$ transitions available to us as diagnostics of electron density, and the larger uncertainties in the measurements in the Fe II $385 \mathrm{~cm}^{-1}$ troughs, we do not consider these differences to be significant.

In contrast, the predicted electron density based on the relative population of the Fe II a ${ }^{4} F_{9 / 2}$ level at $1873 \mathrm{~cm}^{-1}$, being an order of magnitude smaller, does present a possible conundrum. Among all of the column density measurements of transitions from excited states of $\mathrm{Fe}$ II we believe this one to be the most secure. However, the atomic data ( $A$-values and collision strengths) involved in the computations of level populations of the ${ }^{4} F$ term carry greater uncertainties than those pertaining to the levels of the a ${ }^{6} D$ (ground) term. This is because the a ${ }^{4} F$ and a ${ }^{6} D$ terms belong to different electron configurations, i.e., $3 d^{7}$ and $3 d^{6} 4 s$ respectively, and the energy difference between these terms is comparable in magnitude to the relativistic corrections to the Hamiltonian. Thus, the computation of accurate transition rates among levels of these terms requires fine tuning of large atomic configuration expansions (see M. Bautista et al. 2010, in preparation for a more detailed discussion).

We also consider the possibility of some selective excitation process that might lead to a significantly lower population in $\mathrm{Fe}$ II a ${ }^{4} F_{9 / 2}$ than would be inferred by collisions alone. Neither photoexcitation by the continuum radiation field nor

\footnotetext{
9 This situation is present within CLOUDY versions 08 and prior, while future versions are expected to adopt a revised Fe II model (G. J. Ferland 2009, private communication).

${ }_{10}$ We have verified that the effect of the present discrepancy in the atomic data for Fe II on the derived gas density in the main outflow system of SDSS J2359-1241 reported in Korista et al. (2008) is minimal.
} 

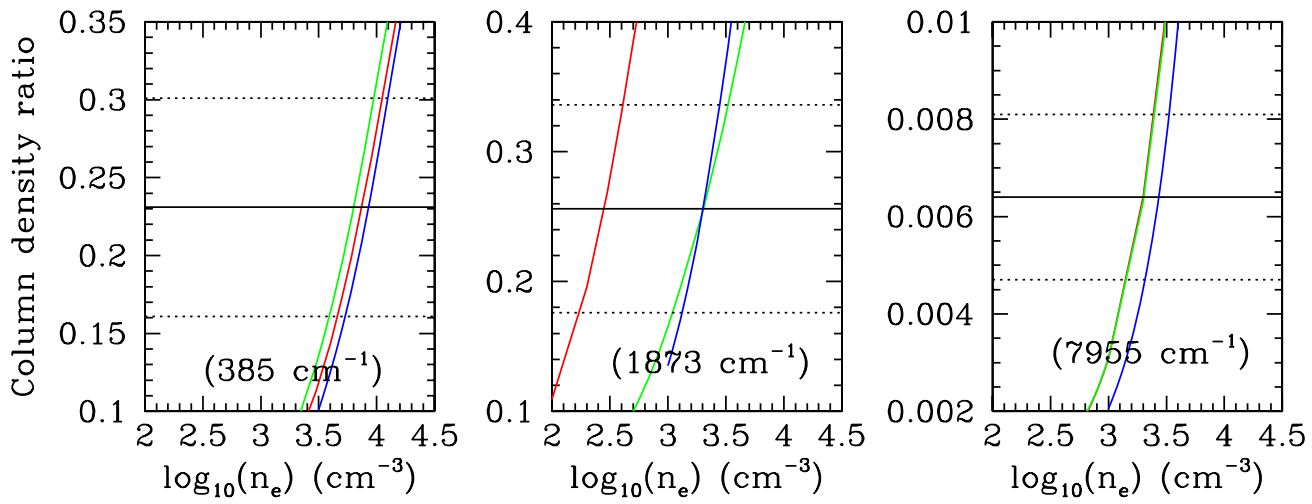

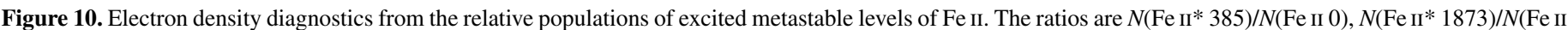

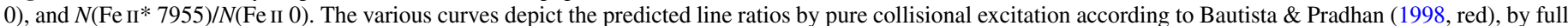

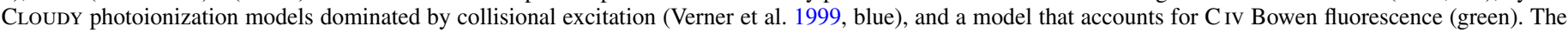

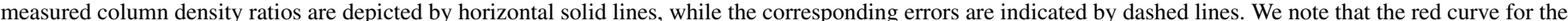
$N\left(\mathrm{Fe}_{\mathrm{II}} * 7955\right) / N(\mathrm{Fe}$ II 0$)$ ratio is located underneath the green line.

Ly $\alpha$ fluorescence can explain the observed discrepancy. Another mechanism, known to occur in spectra of circumstellar envelopes of symbiotic stars and in the environs of gammaray bursts, is Bowen fluorescence of Fe II by $\mathrm{C}$ IV emission at $1548 \AA$ (e.g., Johansson 1983; Eriksson et al. 2008; Vreeswijk et al. 2007; Hartman \& Johansson 2000; Michalitsianos et al. 1992). Pumping occurs as the $1548.19 \AA$ resonance line of C IV closely coincides with the $1548.20 \AA$ transition from the a ${ }^{4} F_{9 / 2}$ metastable level to the ${ }^{4} H_{11 / 2}^{o}$ level of Fe II. Subsequently, this high level cascades down to the ground stage of the ion, so the population of the a ${ }^{4} F_{9 / 2}$ level is re-distributed among other low-lying metastable levels (see also Verner et al. 2000). Fully self-consistent modeling of this fluorescence process requires a detailed treatment of radiative transfer of $\mathrm{C}_{\mathrm{IV}}$ and $\mathrm{Fe}$ II lines within the cloud, which is beyond the scope of this paper. Nevertheless, we can estimate the relative level populations of the observed levels in our spectra under the fluorescence scenario by artificially including its effect in the Bautista \& Pradhan (1998) collisional equilibrium Fe II model. We find that solving this discrepancy requires a photoexcitation rate in the $1548.20 \AA$ transition that is at least 10 times greater than what is available through photoabsorption of radiation from the adopted dereddened SED (Section 4.2). We show the resulting effects on the level populations as the green curves in Figure 10 (i.e., the red curve becomes the green one). Although, this fluorescence mechanism is able to correct the population of the a ${ }^{4} F_{9 / 2}$ level to agree with the observed column density at the same electron density given by $\mathrm{Si}$ II, the pumping rate required seems too large even when considering the inclusion of C IV emission from the broad emission line region. Further investigation of this effect is left for future work. After considering all of these issues pertaining to the various uncertainties, we adopt the Si II-determined electron density of $\log \left(n_{e}\right)=3.3 \pm 0.2\left(\mathrm{~cm}^{-3}\right)$.

Finally, we measure column densities from the ground $3 d^{9}$ ${ }^{2} D_{5 / 2}$ level and the $3 d^{8} 4 s^{4} F_{9 / 2}$ metastable level at $8394 \mathrm{~cm}^{-1}$ for the $\mathrm{Ni}$ II ion. We find that the population of the metastable level exceeds the predictions of pure collisional excitation conditions for any value of the electron density. This is to be expected because Ni II is very efficiently excited by continuum radiation (Bautista et al. 1996; Lucy 1995). As a consequence, Ni II cannot be used as density diagnostic here, but instead serves as a check for the flux of photons below the hydrogen ionization threshold from the central source. As such, Ni II can be used as a direct distance indicator to the outflow (see Section 4.4).

\subsection{The Spectral Energy Distribution}

The SED incident on the outflowing gas has important consequences for the ionization and thermal structures within the gas. In what follows, we provide motivation for our choice of two standard SEDs to use in the photoionization models. While AGNs are routinely observed in the optical/UV and X-rays (see Elvis et al. 1994), the critical EUV spectral region ( $\sim 1$ Ry to $0.1 \mathrm{keV}$, rest frame) is largely inaccessible except near its low- and high-energy extremes. The present working model is that a thermal accretion disk spectrum dominates the energy contained within the continuum spectrum feature known as the "UV bump," sometimes called the "big blue bump" (Shields 1978; Malkan 1983). Such a feature is expected to peak (in units of $v F_{v}$ ) at energies of a few Rydberg, depending on the mass of the SMBH and the mass accretion rate (Shakura \& Syunyaev 1973). Mathews \& Ferland (1987) had this in mind when they constructed a simplified piece-wise power-law representation of a typical QSO SED, basing the relative strength and rollover energy of the UV-FUV bump on measurements of the strengths of the He II broad emission lines (photon counting) and overall energy balance within the broad emission line gas (e.g., comparing the strength of Ly $\alpha 1216$ to the strengths of strong cooling lines such as C IV $\lambda 1549$ ), and joining the turndown onto a power-law representation of the X-ray spectrum for typical X-ray bright quasars (samples of which at that time were dominated by radio loud QSOs). This spectrum, hereafter referred to as MF87, has since been the standard SED in modeling photoionized gas associated with AGNs. We adopt the MF87 SED as one of two standards for our photoionization models.

By the late 1990s, improved UV and X-ray observations of AGNs led several investigators to reconsider the importance of accretion disk emission to the FUV spectra of AGNs. In a sample of luminous QSOs with Hubble Space Telescope (HST) spectra, Zheng et al. (1997) found surprisingly soft continuum SEDs at wavelengths shortward of $\sim 1000 \AA$. Shortly thereafter, Laor et al. (1997) reported that the soft X-ray spectra of quasars appeared to be pointing (in $v F_{v}$ space) at the soft FUV slopes found by Zheng et al. and proposed that a large fraction of the UV bump inferred by Mathews \& Ferland (1987) and standard accretion disk models was missing (see also Elvis et al. 1994). This led to investigations of cooler accretion disk models with thermal spectra that are Comptonized by a $\sim 10^{8} \mathrm{~K}$ plasma, 


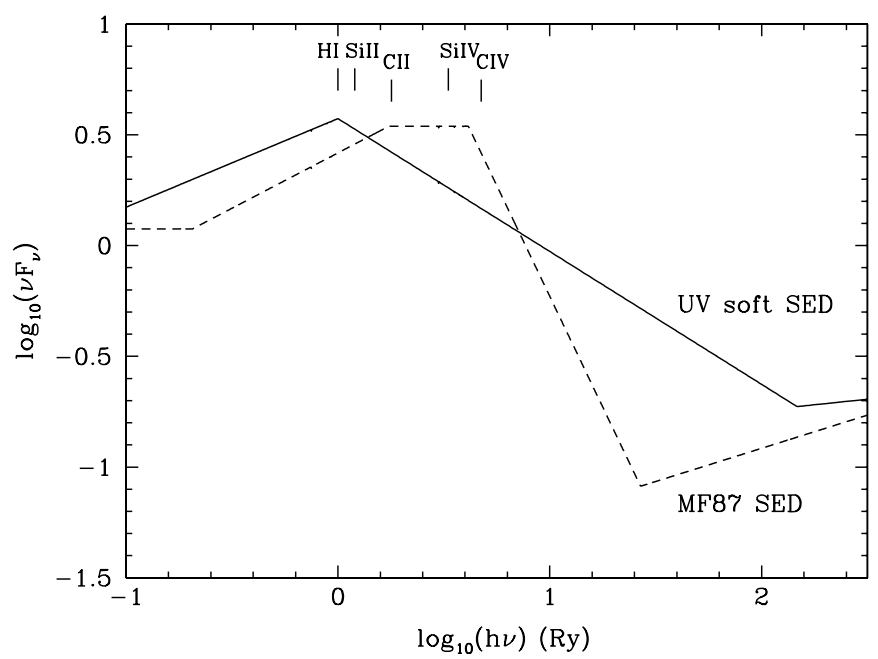

Figure 11. Incident SEDs considered in photoionization modeling. Both SEDs shown have the same bolometric flux. Here, we also mark the ionization energies of some ions of interest (i.e., H I, Si II, Si IV, C II, C IV).

which result in power-law like instead of exponential like high energy tails in the spectrum (e.g., Blaes et al. 2001). Telfer et al. (2002) followed up on the work of Zheng et al. with a larger sample of objects with HST spectra, which improved the statistics at the shorter wavelengths. They found similarly but not quite as soft FUV slopes and concluded for radioquiet quasars "that it is plausible to represent the entire typical ionizing continuum from $\sim 10 \mathrm{eV}$ to $\sim 2 \mathrm{keV}$ by a single power law" $(\alpha \approx-1.6)$. With this in mind Hamann et al. (2001, 2002) constructed a simple piece-wise power law radio-quiet quasar SED to model intrinsic AGN absorption systems and broad emission lines. We adopt a slightly modified version of their SED as our second standard: from $1 \mu \mathrm{m}$ to $1 \mathrm{Ry}, \alpha=-0.6$; from $1 \mathrm{Ry}$ to $2 \mathrm{keV}, \alpha=-1.6$; from $2 \mathrm{keV}$ to $50 \mathrm{keV}, \alpha=-0.9$ and a high-energy break beyond. This SED has an $\alpha_{o x} \approx-1.43$, very nearly equal to that of the $\operatorname{MF87} \operatorname{SED}\left(\alpha_{o x} \approx-1.40\right)$, while effectively excluding the presence of a UV bump peaking at FUV energies. ${ }^{11}$ We refer to this as the "UV-soft SED," and we show it along with the MF87 SED in Figure 11.

With regard to these observational studies of quasar SEDs, the reader should bear in mind that significant uncertainties remain in the corrections in the UV for Galactic extinction (primarily in the adopted value of $R_{V}$ ), in the corrections for atomic absorption within the intervening intergalactic medium (mainly the Ly $\alpha$ forest), as well as in the quasar host galaxy extinction. No attempt was made to correct for the last of these in any of the above works. Thus, from an observational point of view, the existence and magnitude of the $1000 \AA$ break and the apparent roll-down toward the observed X-rays, as suggested in Laor et al. (1997), remain unclear. Scott et al. (2004a), in a sample of Far Ultraviolet Spectroscopic Explorer spectra of luminous Seyfert1 galaxies and low redshift quasars, reported no FUV spectral break down to $\sim 600 \AA$. However, some of the differences between this finding and that in the more luminous AGN sample of Telfer et al. (2002) may be intrinsic, due to differences in the accretion disk physics. We refer the reader to Shang et al. (2005) and Bonning et al. (2007) for recent detailed comparisons between observations and modern accretion disk model predictions, as well as critical evaluations of the current

\footnotetext{
11 Hamann et al. 2001 terminated this power law portion at $1 \mathrm{keV}$, resulting in
} a slightly flatter alpha $o x=-1.35$. uncertainties. We consider it plausible that actual quasar FUV/ EUV SEDs fall somewhere between our two adopted standards.

\subsection{Reddening of the SED}

In the previous section, we constructed a plausible UV-soft SED for SDSS J0318-0600. However, it is known that the UV spectrum of SDSS J0318-0600 is strongly affected by extinction due to dust. Hall et al. (2002) found that if the Small Magellanic Cloud (SMC) reddening curve, typical for QSOs, were adopted, the extinction correction $E(B-V)$ would be between $\sim 0.1$ and $\sim 0.4$ depending on whether the $2000-3000 \AA$ or 1250-2000 $\AA$ rest-frame wavelength ranges were fit to the SDSS composite QSO spectrum. Because the location of the bulk of the reddening of the SED is unknown, we must consider a scenario in which the SED incident on the outflow has been reddened by dust grains.

Figure 12 shows a portion of the SED centered around the restframe energy range of our observations. Here we show the unattenuated UV-soft SED described in the previous section and the FIRST Bright Quasar Survey composite spectrum of Brotherton et al. (2001). The observed spectrum of SDSS J0318-0600, also shown in the figure, is clearly affected by extinction. Five reddened versions of the UV-soft SED are also shown here; two of them are a result of the standard extinction curve of the SMC (Hutchings 1982) with $E(B-V)=0.1$ and 0.4 , and additional curves generated from CLOUDY models of pure silicate grains with a distribution of the form

$$
d n_{\mathrm{gr}}=C n_{H}\left(\frac{a}{a_{0}}\right)^{-\alpha} d a, \quad 20 \AA<a<0.25 \mu \mathrm{m} .
$$

These models were simulated with CLOUDY as the transmitted spectrum of a cloud with a Galactic dust to gas ratio, $\log n_{H}\left(\mathrm{~cm}^{-3}\right)=4.0, \log N_{H}\left(\mathrm{~cm}^{-2}\right)=21.8$ and $\log U_{H}=+2.0$. This high-ionization parameter was chosen in order to minimize atomic features in the resulting spectrum and to allow grain extinction to dominate the attenuation. A cloud of this number density and ionization parameter would be located $\sim 30 \mathrm{pc}$ from the central source roughly with the expected realm of the putative clumpy, dusty torus. The dust temperature in this cloud has a maximum value of $400 \mathrm{~K}$, which is below the sublimation temperature of silicate grains ( $1400 \mathrm{~K}$ for silicates). See Pier \& Voit (1995); Nenkova et al. (2008) for a more extensive discussion of the properties of illuminated dusty tori associated with AGNs. No graphite grains are included in the models because they lead to the $2175 \AA$ peak, which is absent in the spectrum. On the other hand, a large absorption peak from silicates at approximately $690 \AA(\sim 1.3 \mathrm{Ry})$ does correlate with the observed spectrum. The depth of such a peak is dependent on the size distribution of grains (i.e., in the value of $\alpha$ ). A problem arises in determining the values of $\alpha$ and total extinction that are proportional to $C$, because from the UV spectrum alone no unique solution for both values can be found simultaneously. Fortunately, SDSS J0318-0600 was also observed by the Two Micron All Sky Survey (2MASS). The photometric measurements in the $J, H$, and $K$ bands (rest frame 4162, 5602, and $7277 \AA$ respectively) are marked in Figure 12. It is important to note here that the flux in the $J$ band may be affected by $\mathrm{H} \delta$ and other emission lines in the rest frame of the quasar. With this large wavelength range we are able to determine the magnitude of extinction, while the value of $\alpha$ is determined by fitting the slope of the SDSS spectrum. We find $E(B-V)=0.15$ for an extinction curve with a ratio of selective to total extinction $R_{V}=A_{V} / E(B-V)=4.4$ and $\alpha=3.4$. 


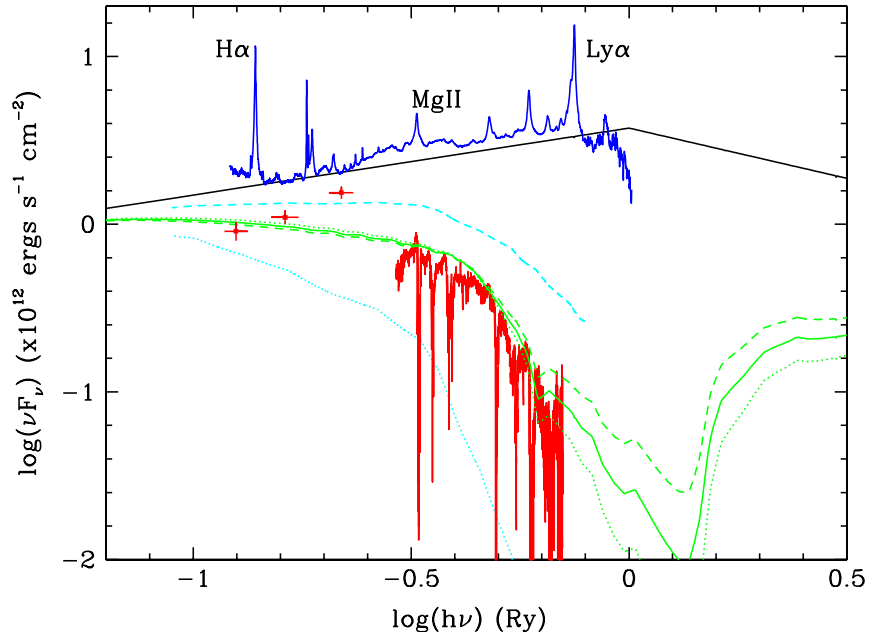

Figure 12. Spectrum of SDSS J0318-0600. The observed SDSS spectrum is depicted by the red curve and the 2MASS $J, H$, and $K$ photometric measurements are indicated by red crosses. The black curve depicts our adopted UV-soft SED and the blue curve shows the FIRST Bright Quasar Survey composite spectrum (Brotherton et al. 2001). The green lines show the reddened UV-soft SED by our distributed silicate grain model with $\alpha=3.4$ (solid line), 3.3 (dotted line), and 3.5 (dashed line). The cyan curves depict the UV-soft SED reddened by the SMC extinction curve with $E(B-V)=0.1$ (dashed line) and 0.4 (dotted line)

The extinction curve of SDSS J0318-0600 with $R_{V}=4.4$ is quite different from that of the SMC with $R_{V} \sim 2$ and the diffuse Galactic Interstellar Medium (ISM) with $R_{V}=3.1$. The steepness of the present curve is more similar to that of dense molecular clouds and star-forming regions with $R_{V} \approx 5$. These large values of $R_{V}$ are characteristic of a distribution of grains that favor larger grains than in typical ISM. This would result if either large grains are allowed to accrete in a dense environment shielded from UV radiation or small grains are efficiently destroyed in a violent event, e.g., shocks. Such may be the environment of the dusty clouds composing the clumpy tori of AGNs.

\subsection{Bolometric Luminosity and Number of Ionizing Photons of SDSS J0318-0600}

Once the extinction that affects the observed spectrum has been determined, we are in a position to compute the bolometric luminosity of the QSO. To do that we adopt the UV-soft SED shown in Figure 12 of the previous section and integrate over the whole energy space. This gives $L_{\mathrm{Bol}}=5.0 \times 10^{47} \mathrm{erg} \mathrm{s}^{-1}$, assuming cosmological parameters of $H_{0}=70 \mathrm{~km} \mathrm{~s}^{-1} \mathrm{Mpc}^{-1}$, $\Omega_{m}=0.3$, and $\Omega_{\Lambda}=0.7$.

With the above normalization and our adopted (UV-soft) SED we find a hydrogen ionizing photon rate of $Q=3.6 \times 10^{57}$ photons $\mathrm{s}^{-1}$. However, it is unclear at this time where most of the extinction occurs with respect to the outflow. If the grain extinction of the SED occurs interior to the outflow, $Q$ should be determined from our preferred dust reddened model $(\alpha=3.4$ and $E(B-V)=0.15)$. For this case, the effective $Q=2.1 \times 10^{56}$ photons s $^{-1}$. We will later use $Q$ in conjunction with the derived values of gas number density and ionization parameter to estimate the distance of the outflow from the central source.

\subsection{Photoionization Modeling}

We start by assuming constant total hydrogen density "clouds" of solar abundances from Lodders (2003). The optimization of all our models is based on the column densities of
Si II and Si IV. This is because they have constrained column density measurements from the spectrum, expand the whole range of ionization stages observed, and they come from the same chemical element, thus their relative strengths are independent of the chemical composition of the cloud.

In trying to reproduce the measured ionic column densities we have constructed a number of photoionization models exploring the physical conditions and our assumptions. For a qualitative description of the ionization structure of the cloud see Korista et al. (2008). It is important in all models that the average electron density predicted by the model coincide with that diagnosed from the Si II lines in Section 4.1 within 0.1 dex. For every model, we compute the electron density averaged over the Si II trough, as

$$
\left\langle n_{e}\right\rangle_{\mathrm{Si} \text { II }}=\frac{\int_{0}^{N_{H}} N_{\mathrm{Si} \mathrm{II}_{\text {II }}} n_{e}\left(N_{H}\right) d N_{H}}{\int_{0}^{N_{H}} N_{\mathrm{Si} \text { II }} d N_{H}} .
$$

This value is presented in Table 7 together with the adopted $n_{H}$.

A summary of the results of different models is presented in Table 7. Here the first three column densities indicate the species observed, their measured column densities, and respective uncertainties. The subsequent column densities present the differences between observations and the results of various models as described below. Here, we also present the optimal $U_{H}$ and $N_{H}$ determined for every model.

We first try to model the cloud using solar abundances and two possible SEDs: our UV-soft SED described above and the UV-soft SED reddened as in the previous section. While the measured silicon column densities can be matched in both cases, this only happens when going very deep into the ionization front where $n_{e}$ and temperature have started to drop. Therefore, we see that in order to reproduce a $\log \left(\left\langle n_{e}\right\rangle\right)=3.3$ in the Si II region, the particle density of the cloud must be $\log \left(\left\langle n_{H}\right\rangle\right)=3.8$. Another concern about these models is their predictions for O I column densities, which come to be about 2 orders of magnitude greater than allowed by observations. These results are shown as models A and B in Table 7 for the unreddened and reddened SEDs, respectively. Because $\mathrm{O}$ I is tightly driven by $\mathrm{H}$ I through charge exchange, the observed column density of $\mathrm{O}_{\mathrm{I}}$ is a direct indicator of the depth of the ionization front with respect to the total column density of the cloud. The same argument is true for the column density of Fe II. The problems with this model are illustrated in Figure 13. Despite the fact that our measurement of $\mathrm{O}_{\mathrm{I}}$ is poor, the discrepancy between this and models with solar composition is significant. The current measured column density is $1.9 \times 10^{15} \mathrm{~cm}^{-2}$ with an uncertainty of a factor of $2-3$. On the other hand, models with solar composition overestimate the column density by 1.9 dex, i.e., $1.5 \times 10^{17} \mathrm{~cm}^{-2}$. This would be the largest column density of all ions in the spectrum, about 5 times that of C IV. Despite the oscillator strength of O I being smaller than that of C IV, a column density as predicted would yield a very strong and completely saturated trough in the spectrum. This is clearly not the case. The fact that the present model goes too deep into the ionization front to reach the high observed ratio of Si II to Si IV column densities indicates that the abundance of $\mathrm{Si}$ relative to $\mathrm{O}$ must be higher than solar.

Similar to $\mathrm{Si}$, the $\mathrm{Fe} / \mathrm{O}$ and $\mathrm{Ni} / \mathrm{O}$ abundance ratios must also be supersolar because the ionic fractions of Fe II, Ni II, and $\mathrm{O}$ I are tied to neutral hydrogen through charge exchange. Thus, the Fe II/O I and Ni II/O I ratios are nearly independent of the physical condition of the cloud and can only be varied by changing the abundances of these elements (Bautista \& Pradhan 
Table 7

Measured and Model Predicted Absorption Column Densities for the Outflow Component i in QSO J0318-0600

\begin{tabular}{|c|c|c|c|c|c|c|c|c|}
\hline Parameter/Ion & $\log (N)\left(\mathrm{cm}^{-2}\right)$ & $\Delta \log (N)$ & A & $\mathrm{B}$ & $\mathrm{C}$ & $\mathrm{D}$ & $\mathrm{E}$ & $\mathrm{F}$ \\
\hline $\log \left(U_{H}\right)$ & & & -2.25 & -2.47 & -2.63 & -3.02 & -2.85 & -3.13 \\
\hline $\log \left(N_{H}\right)$ & & & 20.96 & 20.92 & 20.20 & 20.07 & 19.86 & 19.73 \\
\hline $\log \left(n_{H}\right)$ & & & 3.8 & 3.7 & 3.3 & 3.4 & 3.3 & 3.3 \\
\hline$\left\langle\log \left(n_{e}\right)\right\rangle^{\mathrm{a}}$ & & & 3.3 & 3.3 & 3.3 & 3.3 & 3.3 & 3.3 \\
\hline$\langle\log T\rangle_{\mathrm{Si}_{\text {II }}}$ & & & 4.0 & 4.1 & 3.8 & 4.0 & 3.9 & 3.9 \\
\hline $\log \left(N_{H I}\right)$ & & & 20.50 & 20.43 & 18.50 & 19.36 & 17.48 & 18.26 \\
\hline \multirow[t]{2}{*}{ Ion } & \multicolumn{2}{|c|}{ Observed } & \multicolumn{6}{|c|}{$\log \left(N_{X}\right)_{\text {Predicted }}-\log \left(N_{X}\right)_{\text {Observed }}$} \\
\hline & $\log \left(N_{X}\right)$ & $\Delta \log \left(N_{X}\right)$ & & & & & & \\
\hline Si II & 16.16 & $0.15^{\mathrm{b}}$ & +0.1 & 0.00 & -0.01 & 0.00 & 0.00 & 0.00 \\
\hline Si IV & 15.75 & 0.11 & +0.02 & 0.00 & 0.00 & 0.00 & +0.01 & +0.01 \\
\hline $\mathrm{He} \mathrm{I}^{*}$ & $<14.0$ & & -0.1 & -0.4 & -0.3 & -1.0 & -0.9 & -1.3 \\
\hline $\mathrm{C}_{\text {II }}$ & $>16.3$ & & +0.6 & +0.5 & -0.3 & -0.1 & -0.5 & -0.5 \\
\hline C IV & 16.46 & 0.05 & -0.15 & -0.12 & -1.18 & -1.37 & -1.43 & -1.62 \\
\hline OI & 15.3 & $\begin{array}{l}+0.5 \\
-0.3\end{array}$ & +1.9 & +1.8 & 0.0 & +0.9 & -0.9 & +0.3 \\
\hline $\mathrm{Mg}_{\mathrm{I}}$ & $<12.3$ & & +1.5 & +1.3 & +1.1 & +1.0 & +1.0 & +1.0 \\
\hline $\mathrm{Mg}_{\text {II }}$ & 15.51 & 0.06 & +0.25 & -0.39 & -0.21 & -0.84 & -0.29 & -0.66 \\
\hline $\mathrm{Al}$ II & 14.60 & 0.04 & +0.07 & -0.73 & -0.10 & -0.89 & -0.10 & -0.67 \\
\hline Al III & 15.19 & 0.06 & -0.60 & -1.05 & -0.73 & -1.15 & -0.65 & -1.00 \\
\hline $\mathrm{Fe} \mathrm{III}^{\mathrm{c}}$ & 15.38 & 0.10 & +0.65 & +0.67 & -0.04 & +0.54 & -0.69 & +0.21 \\
\hline $\mathrm{Ni} \mathrm{II}^{\mathrm{c}}$ & 14.36 & $0.30^{\mathrm{b}}$ & +0.45 & +0.49 & +0.04 & +0.46 & -0.57 & +0.23 \\
\hline Zn II & 12.92 & 0.02 & -0.56 & -1.59 & -1.26 & -1.39 & -1.48 & -1.67 \\
\hline
\end{tabular}

Notes. Model A: UV-soft SED and solar abundances; Model B: Reddened UV-soft SED and solar abundances; Model C: UV-soft SED and Ballero et al. (2008) abundances for $Z / Z_{\odot}=4.2$, see Table 8; Model D: Reddened UV-soft SED and Ballero et al. (2008) abundances for $Z / Z_{\odot}=4.2$, see Table 8; Model E: UV-soft SED and Ballero et al. (2008) abundances for $Z / Z_{\odot}=7.2$, see Table 8; Model F: Reddened UV-soft SED and Ballero et al. (2008) abundances for $Z / Z_{\odot}=7.2$, see Table 8 .

${ }^{\text {a }}$ Determined from the population ratio of Si II excited $\left(287 \mathrm{~cm}^{-1}\right)$ to ground levels.

${ }^{\mathrm{b}}$ Column density error dominated by the uncertainty in oscillator strength.

${ }^{\mathrm{c}}$ Calculated from theoretical population balance models from the column densities of observed levels.
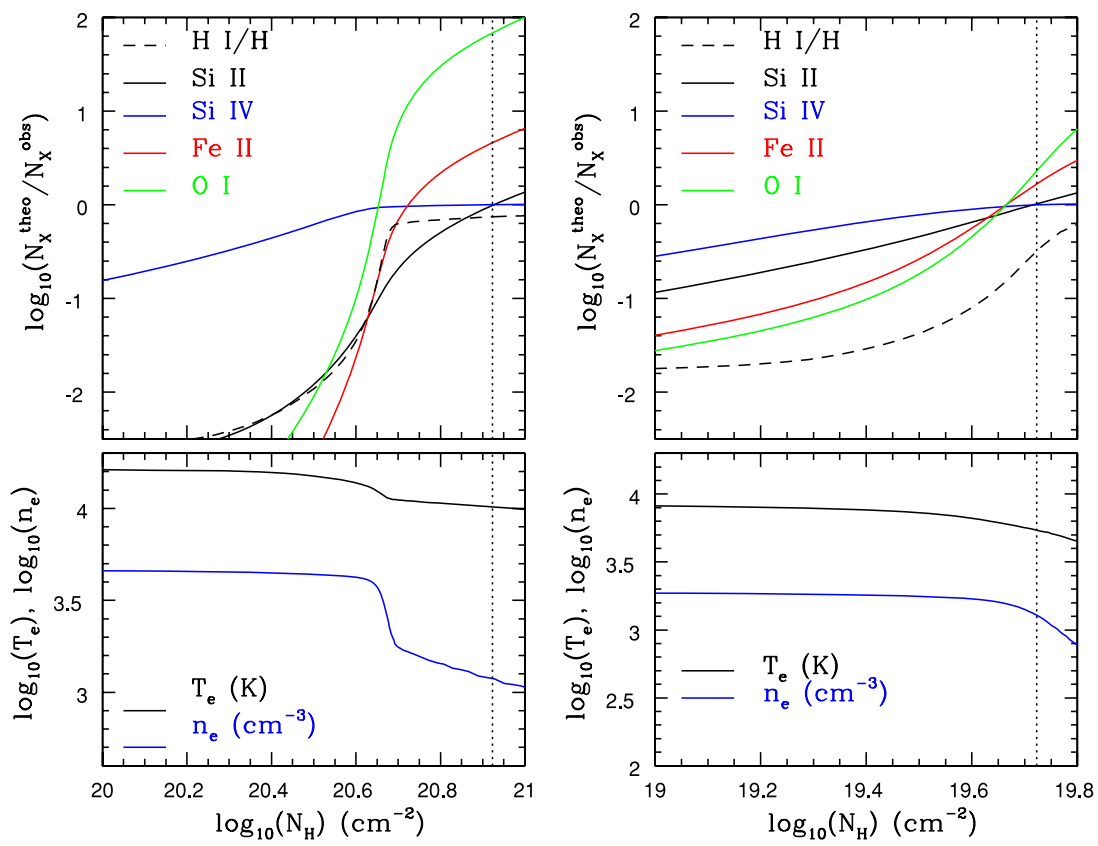

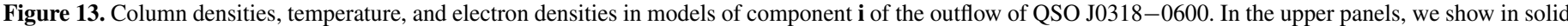

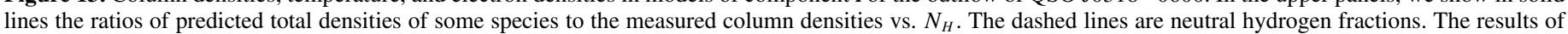

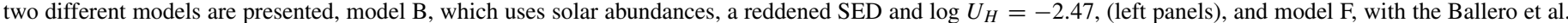

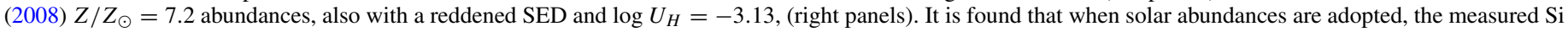
column densities require a total column density that extends beyond the ionization front, which then results in overestimated O I and Fe II column densities.

1998; Bautista et al. 1996). Furthermore, we find that for solar abundances of all other elements, the minimum enhancement of $\mathrm{Si}, \mathrm{Fe}$, and $\mathrm{Ni}$ in gas phase that satisfies the are $[\mathrm{Si} / \mathrm{H}]=+0.8$ and $[\mathrm{Fe} / \mathrm{H}]=[\mathrm{Ni} / \mathrm{H}]=+0.3$.
We note that this abundances pattern is similar to that expected in high-metallicity bulges of galaxies. Ballero et al. (2008) studied the evolution of chemical abundances of spiral bulges hosting Seyfert nuclei by modeling the efficiency of star 
Table 8

Abundances Used for Photoionization Modeling

\begin{tabular}{lcc}
\hline \multicolumn{1}{c}{ Ion } & $Z / Z_{\odot}=4.2$ & $Z / Z_{\odot}=7.2$ \\
\hline$[\mathrm{Fe} / \mathrm{H}]$ & +0.83 & +1.07 \\
{$[\mathrm{O} / \mathrm{H}]$} & +0.18 & +0.48 \\
{$[\mathrm{Mg} / \mathrm{H}]$} & +0.38 & +0.69 \\
{$[\mathrm{Si} / \mathrm{H}]$} & +0.79 & +1.13 \\
{$[\mathrm{Ca} / \mathrm{H}]$} & +0.63 & +1.06 \\
{$[\mathrm{C} / \mathrm{H}]$} & +0.15 & +0.30 \\
{$[\mathrm{~N} / \mathrm{H}]$} & +0.28 & +0.87 \\
\hline
\end{tabular}

formation. They predict the abundances of various elements for $Z / Z_{\odot}=4.23$, 6.11, and 7.22. We present these abundances, which we use for photoionization modeling in Table 8. We present these elemental abundances for the first and last of the metallicities, and use them in the photoionization modeling to investigate the effects of a realistic span in gas metallicity. Ballero et al. (2008) do not predict abundances for Al, which is present in our spectra, thus we simply scale this linearly with $Z$ to $[\mathrm{Al} / \mathrm{H}]=+0.50$ and 0.86 at $Z / Z_{\odot}=4.23$ and 7.22 , respectively. We use the $[\mathrm{He} / \mathrm{H}]$ versus $Z$ relation from those predicted in the chemical evolution models of Hamann \& Ferland (1993). The remaining elements through Zn untabulated by Ballero et al. were assigned abundances as estimated by $Z$.

The results of these models are given as models $C$ through $\mathrm{F}$ in Table 7. It can be seen that these abundances yield better agreement with respect to the measured column densities than when solar abundances are used. Moreover, the abundance enhancements for $Z / Z_{\odot}=4.23$ still yield overestimated column densities for $\mathrm{O}$ I by one to two sigma, depending on the SED used. On the other hand, the models with abundances for $Z / Z_{\odot}=7.2$ agree well in terms of the $\mathrm{O}_{\mathrm{I}}$ predictions if the reddened UV-soft SED is adopted, but underestimate the O I column density when the non-extinguished UV-soft SED is used.

Given our arguments for metallicities greater than solar in the outflow and the fact that the solar models are unable to match the $n_{H}$, the true metallicity in QSO J0318-0600 is likely to be several times solar. Regardless of this we are able to well constrain $U_{H}$ and $N_{H}$. Models $\mathrm{C}$ and E constrain $\log \left(U_{H}\right)$ to -2.75 and -2.85 and $\log \left(N_{H}\right)$ to 20.16 and $19.86\left(\mathrm{~cm}^{-2}\right)$, respectively, for the unreddened SED. While, models D and $\mathrm{F}$ constrain $\log \left(U_{H}\right)$ to -3.02 and -3.13 and $\log \left(N_{H}\right)$ to 20.07 and $19.73\left(\mathrm{~cm}^{-2}\right)$, respectively, for the reddened SED.

One significant discrepancy between all models and observations is in the carbon column densities. As indicated in Table 7, the $[\mathrm{Si} / \mathrm{C}]$ ratio is rather large in these two elemental abundance models, and a relatively larger carbon abundance would reduce the discrepancies. Ballero et al. point out that their prediction for $\mathrm{C}$ is likely uncertain as it depends sensitively on the mass of the bulge. However, it is important to point out that our measurements for carbon are problematic, as we only have a lower limit for the $\mathrm{C}$ II column density and large portions of the $\mathrm{C}$ IV troughs are highly saturated.

A relatively small uncertainty in $U_{H}$ and $N_{H}$ arises from the adopted standard SED. For example, should we adopt the MF87 SED rather than the UV-soft SED constructed in Section 4.2, our results for $U_{H}$ and $N_{H}$ would be affected at a $\sim 0.1$ level. However, this effect is very small compared to the uncertainties resulting from the dust extinction of the SED and the adopted chemical abundances.

Another discrepancy in the models stems from the predictions for $\mathrm{Al}$. Most of our models underestimate the column densities of $\mathrm{Al}$ II and $\mathrm{Al}$ III. The predicted column density ratios of $\mathrm{Al}$ II to Al III differ from observations by approximately a factor of 4. Again, it could be that the true $\mathrm{Al}$ abundance is higher than assumed, which is not surprising as there are only very rough approximations on how this element scales with metallicity. But, beyond the absolute column densities there may also be differences in the predicted ionization structure of Al. The other problem is that the predicted column densities of Mg I always exceed the upper levels derived from our spectra. It is clear that the ionization structure of neutrals that form by recombination across the ionization front is not well described by our models. The cause for this problem is a combination of inaccuracies in the atomic parameters for these species and limitations of photoionization modeling codes to treat neutral ions with lowionization potential $(<10.2 \mathrm{eV})$. The exception to this is $\mathrm{He} \mathrm{I}^{*}$, which is consistent with the models predictions. This problem is intrinsic to all modeling codes that treat radiative transfer through the escape probability approximation, as neutral species with low-ionization potential are greatly affected by Lyman $\alpha$ radiation. Thus, a very accurate treatment of radiative transfer is needed to confidently model these neutral species, which is beyond the scope of this paper.

\subsection{The Ni II Lines as Independent Distance Indicators}

Lucy (1995) demonstrated that Ni II could be efficiently photo-excited by continuum radiation under nebular conditions. Bautista et al. (1996) constructed a detailed 142 level model of the Ni II system for spectral analysis, which was updated with improved atomic data by Bautista (2004).

Figure 14 shows the level populations ratio between the excited level at $8394 \mathrm{~cm}^{-1}$ and the ground level. The upper panel shows the ratio versus $n_{e}$ under pure collisional excitation conditions. It is noted that the uncertainty in the measured ratio column densities is dominated by that in the oscillator strengths, particularly for troughs from the $8394 \mathrm{~cm}^{-1}$. For these transitions, we adopted oscillator strengths from Kurucz \& Bell (1995), which in the case of the resonant transitions were discrepant by up to a factor of 2 from experimental determinations of Fedchak \& Lawler (1999). Thus, we adopt a nominal $30 \%$ uncertainty in the observed ratios. Further theoretical and experimental work on the oscillator strengths of Ni II is warranted.

From Figure 14 one sees that the observed ratio is consistent with the predictions of pure collisional excitation for a wide range in $n_{e}$ that includes the conditions of the absorbed as diagnosed from Si II. Hence, it should be possible to set an upper limit to the continuum flux that also contributes to the excitation of the $8394 \mathrm{~cm}^{-1}$ level. In the lower panel of the figure, we plot the ratio against the flux of ionizing photons $\Phi$ for the same SED used in our photoionization models. It is important to note that only photons less energetic than 1 Ry participate in photoexcitation of the ion and these are unaffected by the main atomic opacities of the cloud. It is found that the $\mathrm{N}(\mathrm{Ni}$ II 8394$) / \mathrm{N}(\mathrm{Ni}$ II 0$)$ ratio is strongly enhanced by continuum fluorescence and this drives the ratio well above the Boltzman equilibrium populations. Under these conditions, induced photoexcitation of highly excited odd parity levels is balanced by rapid spontaneous radiative decay. For much more intense radiation fields, induced photoexcitation exceeds spontaneous decay and the level population ratio reaches a plateau. It can be seen that the observed column density ratio sets upper limits of $\log _{10}(\Phi / c) \approx+1.5$ or +0.4 depending on whether one uses a reddened or unreddened SED. Thus, from a 

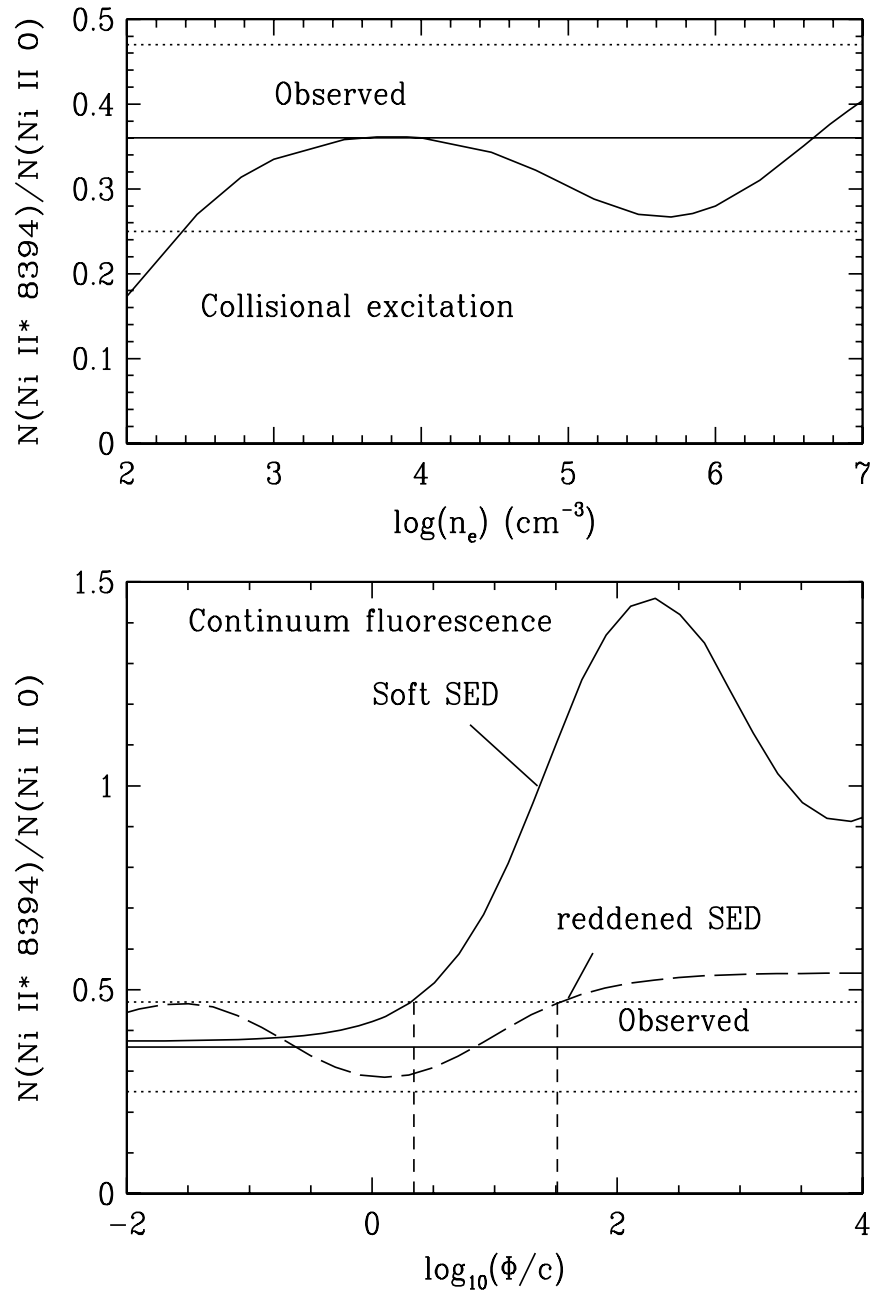

Figure 14. Ratio of level populations between the Ni II excited level at the $3 d^{8} 4 \mathrm{~s}{ }^{4} F_{9 / 2}$ level at $8394 \mathrm{~cm}^{-1}$ and the ground level. The ratio is plotted against $\log \left(n_{e}\right)$ for pure collisional excitation (upper panel) and $\log (\Phi / c)$ for continuum fluorescence excitation. The observed ratio is depicted by the solid horizontal line and the uncertainties are shown by dotted lines and the vertical dashed lines represent the upper limits for $\log _{10}(\Phi / c)$ as constrained by the measured ratio.

total particle density of $\log \left(n_{H}\right)=3.3$ one derives a value for the ionization parameter $\log _{10}\left(U_{H}\right) \leqslant-1.8$ or $\leqslant-2.9$, for reddened and unreddened SEDs respectively. Both of these values are consistent with that derived through photoionization modeling. It is unfortunate that more stringent constrains could not be derived due to the large uncertainties in the oscillator strengths for the absorption lines in the spectrum.

\section{KINETIC LUMINOSITY AND MASS FLUX OF THE OUTFLOW}

\subsection{Calculating $\dot{M}$ and $\dot{E}_{k}$ for the Main Outflow Component}

In the previous section, we determined the ionization parameter, number density, and total column density of the main outflow component, (i). Here, we calculate the mass and energetic characteristics of the outflow. First, we determine the mass contained within the wind. For simplicity, we assume that the outflow is in the form of a partial thin spherical shell at a distance $R$ from the central source moving radially at constant speed $v$. The total mass contained in such an outflow is

$$
M=4 \pi \mu m_{p} \Omega R^{2} N_{H},
$$

where $N_{H}$ is the total column density of hydrogen, $m_{p}$ is the mass of a proton, $\mu=1.4$ is the mean molecular weight of the plasma per proton, and $\Omega$ is the fraction of the shell occupied by the outflow. Therefore, assuming $\mathrm{d} N_{H} / \mathrm{d} t=0$, the mass flux follows as the temporal derivative:

$$
\dot{M}=8 \pi \mu m_{p} \Omega R N_{H} v,
$$

the momentum flux is given by

$$
\dot{p}=\dot{M} v,
$$

and the kinetic luminosity is given by

$$
\dot{E}_{k}=\frac{\dot{M} v^{2}}{2}=4 \pi \mu m_{p} \Omega R N_{H} v^{3} .
$$

For component i: $v=-4200 \mathrm{~km} \mathrm{~s}^{-1}$ and Table 6 gives the $N_{H}$ for each of our photoionization models. To calculate the distance $R$ we use Equation (4), where $n_{e}$ is determined in Section 4.1, $U_{H}$ is taken from Table 6 and $Q_{H}$ is given in Section 4.4, $\log Q_{H}$ (photons $\mathrm{s}^{-1}$ ) as 56.3 and 57.6 photons $\mathrm{s}^{-1}$ for the attenuated and unattenuated SEDs, respectively. In Table 9, we list the derived values for $R, \dot{E}_{k}$, and $\dot{M}$ for each photoionization model.

\subsection{The Global Covering Fraction}

As shown above, we determined $N_{H}$ and $R$ for the photoionization models and therefore each model is associated with a reliable estimate of the kinetic luminosity per unit solid angle. In order to obtain estimates for the total kinetic luminosity, we need to address the remaining variable in Equation (1), $\Omega$, the fraction of the full sphere occupied by the outflow. There is no direct way to obtain the $\Omega$ of a given outflow from its spectrum as we only see the material along the LOS. Statistically, C IV BALs are seen in $20 \%$ of all quasars (Hewett \& Foltz 2003). This can be interpreted as (1) all quasars have BAL winds covering $20 \%$ of the solid angle, (2) $20 \%$ of all quasars have winds covering the full solid angle, or (3) all quasars have a phase of full solid angle wind lasting $20 \%$ of their duty cycle. A combination of these three options is also possible. It is important to note that with all else equal, options (1) and (3) yield the same integrated mass flux and kinetic luminosity over the lifetime of an individual quasar, and that all three options yield similar integrated quantities for a sample of quasars.

However, we cannot measure distances to the majority of C IV outflows, but only to those outflows that show troughs from excited and metastable levels. For most of our objects (including SDSS J0318-0600), the spectra cover such transitions only from singly ionized species (e.g., Fe II, Si II, C II, and Ni II). Outflows with troughs from these ions are rare (Trump et al. 2006). Our comprehensive search of the 50,000 brightest SDSS quasars yielded 100 such outflows. We found that only $~ 1 \%$ of the objects that show a CIV outflow show troughs from these singly ionized species. We therefore have two limits to explore with regard to the $\Omega$ of the outflow. First, outflows that exhibit troughs from these four singly ionized species excited states may represent all regular C IV outflows viewed through special conditions that allow for the formation of singly ionized troughs. This scenario yields $\Omega \simeq 0.2$ as is the case for the frequency of CIV BALQSOs among all quasars. The second limit is $\Omega \simeq 0.002$, which is the minimal covering fraction needed to explain the 100 occurrences in 50,000 objects.

Several arguments suggest that the first scenario is closer to the actual physical situation. Since outflows with troughs from 
Table 9

Calculated Values for Each Photoionization Model

\begin{tabular}{lcccccc}
\hline \multicolumn{1}{c}{ Parameter } & \multicolumn{3}{c}{ Unattenuated SED } & \multicolumn{3}{c}{ Attenuated SED } \\
\hline Abundances & Solar & $4.2^{\mathrm{a}}$ & $7.2^{\mathrm{b}}$ & Solar & $4.2^{\mathrm{a}}$ & $7.2^{\mathrm{b}}$ \\
Model & $\mathrm{A}$ & $\mathrm{C}$ & $\mathrm{E}$ & $\mathrm{B}$ & $\mathrm{D}$ & $\mathrm{F}$ \\
$\log U_{H}$ & -2.25 & -2.63 & -2.85 & -2.47 & -3.02 & -3.13 \\
$\log N_{H}\left(\mathrm{~cm}^{-2}\right)$ & 20.96 & 20.20 & 19.86 & 20.92 & 20.07 & 19.73 \\
$R(\mathrm{kpc})$ & 9.4 & 14.6 & 18.7 & 2.9 & 5.5 & 6.3 \\
$\dot{E}_{k}\left(\Omega_{0.2} 10^{45} \mathrm{erg} \mathrm{s}^{-1}\right)^{\mathrm{c}}$ & 12 & 3.1 & 1.8 & 3.3 & 0.9 & 0.5 \\
$\dot{E}_{k} / L_{\mathrm{Bol}}\left(\Omega_{0.2}\right)$ & 0.02 & 0.006 & 0.004 & 0.007 & 0.002 & 0.001 \\
$\dot{M}\left(\Omega_{0.2} M_{\odot} \mathrm{yr}^{-1}\right)$ & 2080 & 560 & 330 & 590 & 160 & 80 \\
\hline
\end{tabular}

Notes.

${ }^{\text {a }}$ Ballero et al. (2008) abundances for $Z / Z_{\odot}=4.2$.

b Ballero et al. (2008) abundances for $Z / Z_{\odot}=7.2$.

${ }^{\mathrm{c}} \Omega_{0.2} \equiv \Omega / 0.2$.

singly ionized species are rare, we may simply be observationally biased by this low-ionization selection effect as we lack excited and metastable level troughs from C IV or species with similar ionization. One physically motivated picture is that we are looking at a normal CIV outflow through a LOS passing through semi-opaque material at the edge of the putative AGN torus. Such a scenario explains why we see the singly ionized species (lowering of the ionization parameter); why most of the objects we measure have strong dust reddening (torus dust) and why these systems are so rare (a solid angle that has sufficient torus material to lower the ionization parameter significantly but not enough to make it a type II quasar).

Recent observations by K. Aoki et al. (2010, in preparation) give support to the above scenario. A quasar showing strong Fe II absorption troughs and strong dust reddning in 2002 showed much less dust extinction and very little Fe II absorption in 2008. Thus, the amount of dust along our LOS changed drastically between two epochs separated by $\sim 3.5$ years in the rest frame of the source. The interpretation is that the dust source has vacated the LOS, therefore increasing the ionization parameter of the outflow and reducing the column density of Fe II. A 3.5 year timescale favors changes in a near-by obscuring material (i.e., the putative torus) over material at a $\mathrm{kpc}$ scale and therefore supports both of our assertions: (1) that the dusty material seen in the spectra of these outflows is indeed much closer to the source than the outflows themselves; (2) that these excited-state singly ionized absorption outflows are naturally explained by a special LOS grazing the putative torus.

Further support for this scenario comes from the fact that all our objects show a CIV BAL, which is morphologically indistinguishable from $\mathrm{C}_{\mathrm{IV}}$ BALs in the ubiquitous high ionization BALQSOs. We therefore conclude that it is more likely that we are seeing normal $\Omega \simeq 0.2$ outflows through a rare LOS.

A direct resolution of this issue necessitates imaging of the winds. This is a difficult task due to the high redshifts of the objects and the presence of a very bright quasar at the center of the image. Nonetheless, we hope to pursue this line of research in the future. Another approach is to observe at wavelengths shortward of $L y \alpha$ where there are a few excited state troughs from higher ionization species. In particular S IV/S IV* $\lambda \lambda 1062.66,1072.97$. This ion is similar to $\mathrm{C}$ IV in ionization structure and indeed S IV troughs are much more prevalent than those of the singly ionized species. Measuring $N_{H}$ and $R$ for these objects will therefore eliminate the uncertainty connected with the rarity of outflows showing troughs from singly ionized species, and will yield robust measurements for
$\dot{M}$ and $\dot{E}_{k}$. We are currently pursuing this project, which is challenging due to the heavy Ly $\alpha$ forest contamination.

\subsection{The Super Massive Black Hole and Host Galaxy}

Given the bolometric luminosity and assuming that the SMBH is accreting near the Eddington limit we find that the amount of mass being accreted is

$$
\dot{M}_{\mathrm{acc}}=\frac{L_{\mathrm{Bol}}}{\epsilon_{r} c 2}=88 M_{\odot} \mathrm{yr}^{-1},
$$

where we assumed an accretion efficiency $\left(\epsilon_{r}\right)$ of 0.1 (Salpeter 1964 ) and $c$ is the speed of light. This large accretion rate (the outcome of a very luminous quasar) suggests that the mass flux values we derive (see Table 8) are not unreasonable. We expect such an outflow to have roughly an order of magnitude higher mass flux than the accretion rate onto the SMBH since the original outflow entrained material on its journey to several $\mathrm{kpc}$ distance. Simple momentum conservation requires that an outflow which started at $\sim 20,000-30,000 \mathrm{~km} \mathrm{~s}^{-1}$ have 5-7 times more mass flux by the time it slowed to $4200 \mathrm{~km} \mathrm{~s}^{-1}$. Therefore, an outflow such as this could have begun with $\dot{M}_{\text {outflow }} \sim \dot{M}_{\text {acc. }}$.

Next, assuming Eddington accretion, we can derive the SMBH mass and infer a mass for the host galaxy bulge. The black hole mass derived from Eddington accretion is (Peterson 1997, p. 238)

$$
M_{\mathrm{BH}}=\frac{L_{\mathrm{Bol}} \sigma_{T}}{4 \pi G m_{p} c}=4.0 \times 10^{9} M_{\odot},
$$

where $\sigma_{T}$ is the Thompson scattering cross-section for an electron, $G$ is the gravitational constant, $M_{\mathrm{BH}}$ is the mass of the SMBH, and $m_{p}$ is the mass of the proton. Thus, the spheroidal bulge component of the host galaxy (assumed to be approximately the mass of the host galaxy) has a mass $M_{\text {bulge }}=3.3 \times 10^{12} M_{\odot}$ based on a SMBH to bulge ratio of 0.0012 (McLure \& Dunlop 2002). This mass can be compared with the total mass ejected by the outflow over the $\sim 10^{8}$ year duty cycle of the quasar, which is a few times $10^{10} M_{\odot}$.

\section{DISCUSSION}

In this section, we compare the results from this object to other outflows found in the literature; discuss the location of the dust in SDSS J0318-0600, which is a major cause for uncertainty in the derived mass flux and kinetic luminosity; elaborate on the relationship between the dynamical timescale of the outflow 
Table 10

Properties of Measured Outflows to Date

\begin{tabular}{|c|c|c|c|c|c|c|}
\hline Object & $\begin{array}{c}R^{\mathrm{a}} \\
(\mathrm{kpc})\end{array}$ & $\begin{array}{l}\log N_{H} \\
\left(\mathrm{~cm}^{-2}\right) \\
\end{array}$ & $\log \mathrm{U}_{H}$ & $\begin{array}{c}\log \dot{E}_{k} \\
\left(\operatorname{erg~s}^{-1}\right)\end{array}$ & $\begin{array}{c}\dot{M} \\
\left(M_{\odot} \mathrm{yr}^{-1}\right)\end{array}$ & Reference $^{b}$ \\
\hline QSO 0059-2735 & $0.001-0.05$ & $\gtrsim 21.5^{\mathrm{c}}$ & -0.7 & $\gtrsim 41.1-42.8$ & $\gtrsim 0.2$ & 1 \\
\hline 3C 191 & 28 & 20.3 & -2.8 & 44.0 & 310 & 2 \\
\hline QSO 1044+3656 & $0.1-2.1$ & $20.0-22.0$ & -1.0 to -6.0 & $44.5-45.4$ & $74-530$ & 3 \\
\hline FIRST 1214+2803 & $0.001-0.03$ & $21.4-22.2$ & -2.0 to -0.7 & $41.6-43.8$ & $0.3-55$ & 4 \\
\hline FIRST $0840+3633$ & 0.001 & $\sim 21.3$ & $<-1.8$ & $>41.9$ & $>0.3$ & 5 \\
\hline FIRST $0840+3633^{d}$ & 0.23 & $\cdots$ & $\cdots$ & $\cdots$ & $\cdots$ & 5 \\
\hline QSO 2359-1241 & 3 & 20.6 & -2.4 & 43.7 & 93 & 6 \\
\hline SDSS J0838+2955 & 3.3 & 20.8 & -1.9 & 45.7 & 590 & 7 \\
\hline SDSS J0318-0600 & 6 or 17 & 19.9 or 20.0 & -3.1 or -2.7 & 44.8 or 45.4 & 120 or 450 & 8 \\
\hline
\end{tabular}

Notes.

${ }^{\text {a }}$ For relative accuracies, see Section 1.

b (1) Wampler et al. (1995), (2) Hamann et al. (2001), (3) de Kool et al. (2001), (4) de Kool et al. (2002a), (5) de Kool

et al. (2002b), (6) Korista et al. (2008), (7) Moe et al. (2009), (8) This work.

${ }^{c}$ Based on Table 5 in Wampler et al. (1995).

d Distance derived from Fe II fluorescence and no photoionization modeling was performed for this object.

and the duty cycle of the quasar; and conclude by describing the possible role of these outflows in AGN feedback phenomena.

In Table 10, we compare all the analyses in the literature that can be used to obtain mass flux and kinetic luminosity for outflows in individual quasars. We also note that with the exception of SDSS J0318-0600, every absorber shows evidence for inhomogeneous LOS covering. For outflow absorption, we refer to homogeneity as the spatial distribution of gas across our LOS to the quasar (see Section 3.2), where a homogeneous flow would uniformly cover the background emission source (LOS covering a factor of 1). Initially, this may be attributed to the large distance of the absorber; however, the outflow in 3C 191 shows evidence of inhomogeneous covering at a greater distance than we find component $\mathbf{i}$ of SDSS J0318-0600.

SDSS J0318-0600 is also the most heavily reddened in the sample, which leaves us with the question of the location of the obscuring dust. Based on the value from Schlegel et al. (1998), we know that the Milky Way Galactic reddening is minimal, and thus the reddening is intrinsic to the quasar. There exist three possible locations of the dust with respect to the absorber: interior, dispersed within, and exterior. The two locations for the dust we consider here due to their potential impact on the modeling of the outflow are that the dust is interior to the absorber or the dust is associated with the absorber. If the dust is interior to the absorber, a plausible source of dust would be the edge of the putative obscuring torus of the AGN unified model (Antonucci \& Miller 1985). This is supported by the object discussed by K. Aoki et al. (2010, in preparation) and furthermore would provide the rare sight line needed to match the frequency of detections for low-ionization BAL outflows that show iron absorption (FeLoBAL), as discussed in Section 5.1. By contrast, galactic wind imaging in nearby galaxies shows that dust co-exists inside the winds and may be associated with the outflows (e.g., M82; Ichikawa et al. 1994). Elvis et al. (2002) proposed the possibility of grains forming within AGN outflows, as conditions allowed. We note though that if the outflowing gas contains the observed dust, the amount of dust depletion must be substantially smaller than in the ISM or SMC in order to produce the observe ionic column densities. Therefore, while we cannot strongly exclude the possibility of a dusty outflow, locating the dust at the edge of the putative torus is physically more plausible and explains several observed features in the FeLoBAL population: the heavy reddening of FeLoBALs, the low reddening experienced by $\mathrm{C}$ IV BAL quasar, and the higher prevalence of high ionization BALSQSOs compared with FeLoBALs.

The dynamical time for component $\mathbf{i}$ of the outflow is $t_{d}=R / v \approx 10^{6} \mathrm{yr}$ (assuming $R=6 \mathrm{kpc}$ ). Therefore, if we are seeing a representative stage of the outflow, over its $\sim 10^{8} \mathrm{yr}$ duty cycle (Hopkins et al. 2005b), the quasar must eject roughly hundred times more mass than seen in component i. Obvious candidates for more ejected mass are the other 10 components of the outflow. However, M. Bautista et al. (2010, in preparation) show that these components collectively do not contribute appreciably to the total mass output for SDSS J0318-0600. Earlier episodes of mass ejection may not be detectable in absorption. Let us assume that component $\mathbf{i}$ is continuing at a constant velocity and no longer entraining any significant mass. Within $10^{7} \mathrm{yr}$, its distance will increase by a factor of 10 and therefore its optical depth will drop by a factor of 100, making it undetectable in absorption. To complete the picture we note that in addition to the FeLoBAL system, SDSS J0318-0600 has a high-ionization BAL at $\sim 27000 \mathrm{~km} \mathrm{~s}^{-1}$. This outflow component is probably much closer and therefore represents a very young mass ejection episode that in time may evolve to something resembling component $\mathbf{i}$. Similar situation exists in other objects (e.g., SDSS J0838+2955; Moe et al. 2009).

Given these caveats the kinetic luminosity, such as the one we have measured in SDSS J0318-0600, provides $\sim(0.1-$ $0.6) \Omega_{0.2} \%$ of the bolometric luminosity as feedback. We note that the measured mass of component $\mathbf{i}$ represents only the cold phase $\left(T=10^{4} \mathrm{~K}\right)$ of the outflow. The hot phase of the gas (or $\mathrm{X}$-ray, $T=10^{6} \mathrm{~K}$ ) has been shown in Seyfert galaxies (e.g., NGC 3783; Netzer et al. 2003) to contribute up to $50 \times$ more column density than the cold phase, therefore it is possible that the gas in the hot phase of the outflow in SDSS J0318-0600 could contribute significantly to the mass flux and kinetic luminosity. For comparison, AGN feedback models of galaxy growth and evolution require a mechanical energy input of roughly $5 \%$ of the bolometric luminosity of the quasar. For example, Di Matteo et al. (2005) use this ratio to recreate the observed $\mathrm{M}_{\mathrm{BH}}-\sigma$ relation and Hopkins et al. (2005a) show in their models that the same value is required to "uncover" a shrouded quasar after a merger. Thus, the absorption outflow we 
observe in SDSS J0318-0600 can be a significant contributor for AGN feedback processes.

Beyond the effects of feedback on the host galaxy, if SDSS J0318-0600 is representative of the type of quasars found in clusters, then we also see that an outflow with the kinetic luminosity of SDSS J0318-0600 would contribute energy to the gas that forms the ICM. The models of Scannapieco \& Oh (2004) show that a 5\% feedback ratio would suffice to heat the ICM during formation of the cluster and provide the well-known $L_{x}-T$ relationship (Kaiser 1986; Arnaud \& Evrard 1999) Also, related to clusters are X-ray cavities in the ICM, which coincide with relativistic radio jets (e.g., NGC1275 in the Perseus cluster, Fabian et al. 2006). We suggest that the sub-relativistic outflows could be related to the bubbles or perhaps are the driving mechanisms for bubble inflation. This follows because the inflation of a bubble is a simpler process provided a wide angle wind (Sternberg et al. 2007). Rafferty et al. (2006) show that the power needed to inflate these bubbles is $\sim 10^{45} \mathrm{erg} \mathrm{s}^{-1}$, which is similar to the kinetic luminosity we measure in SDSS J0318-0600 of $\sim(1-3) \times 10^{45} \mathrm{erg} \mathrm{s}^{-1}$. These similarities in fraction of the bolometric luminosity and power output suggest that quasar outflows play significant roles in influencing their host galaxy and neighborhoods through feedback.

\section{SUMMARY}

1. We present $6.2 \mathrm{hr}$ of VLT/UVES high-resolution spectroscopic observations of the BAL quasar SDSS J0318-0600.

2 . Using the Al II $\lambda 1671$ trough, we divide the outflow into 11 kinematic components ranging in velocity from -7400 to $-2800 \mathrm{~km} \mathrm{~s}^{-1}$. We utilize these components to identify the corresponding absorption troughs in several lines from other ions.

3. For three of these components, we measure many ionic column densities and develop methods to estimate ionic column densities in blended and saturated regions. The Al III doublet data show that all 11 components follow the expected doublet ratio of 2:1 in optical depth and therefore the covering of the emission source is homogeneous. Further tests for components $\mathbf{i}$ and $\mathbf{k}$ with Fe II resonance lines demonstrate the same result.

4. The measured column densities of $\mathrm{Si}$ II and $\mathrm{Si} \mathrm{II}^{*}$ allow us to determine a number density of $10^{3.3} \mathrm{~cm}^{-3}$ for component i, which is the main and innermost component.

5. We show that the column density ratio of Ni II excited to resonance can be used to independently determine the flux of non-ionizing photons, which when combined with the assumed SED yields a direct indication of the distance. These values agree with that derived from Si II.

6. We analyze the intrinsically reddened spectrum of SDSS J0318-0600 and find that it requires a different extinction curve from the known SMC curve. We fit the spectrum with a reddened SED due to pure silicate dust and a power law grain size distribution with a power of $\alpha=3.4$, which resulted in an $R_{V}=4.4$. Based on our model of the intrinsically reddened SED, we estimate the bolometric luminosity $\left(\log L_{\mathrm{Bol}}=47.7\right)$ and the number of ionizing photons illuminating the absorber with and without dust extinction $(\log Q=56.3$ or 57.6$)$.

7. For component i, our photoionization models suggest supersolar abundances. The output total hydrogen column density and the so-called ionization parameter for both cases with and without dust extinction are $N_{H}=10^{19.9}$ or $10^{20.0} \mathrm{~cm}^{-1}$ and $\log U_{H}=-3.1$ or -2.7 respectively.
8. Given the gas density and ionization parameter, and an estimate of the source luminosity, we determine the distance to the outflow for attenuated and unattenuated SEDs of 5.9 or $17 \mathrm{kpc}$ respectively and determine the corresponding kinetic luminosities to be $(0.7$ or 2.5$) \times 10^{45} \Omega_{0.2} \mathrm{erg} \mathrm{s}^{-1}$, and the mass fluxes to be (120 or 450) $\Omega_{0.2} M_{\odot} \mathrm{yr}^{-1}$. We find that component $\mathbf{i}$ is the dominant source of energy and mass when compared to the other kinematic components, which are evaluated in M. Bautista et al. (2010, in preparation).

9. The ratio $\dot{E}_{k} / L_{\mathrm{Bol}}=(0.1-0.6) \Omega_{0.2} \%$ for component $\mathbf{i}$ in SDSS J0318-0600 makes this outflow a significant contributor to AGN feedback mechanisms.

We acknowledge support from NSF grant AST 0507772 and from NASA LTSA grant NAG5-12867. M.A.B. received partial support for this work from the NASA Award No. NNX09AB99G.

\section{REFERENCES}

Adelman-McCarthy, J. K., et al. 2008, ApJS, 175, 297

Antonucci, R. R. J., \& Miller, J. S. 1985, ApJ, 297, 621

Arav, N., Becker, R. H., Laurent-Muehleisen, S. A., Gregg, M. D., White, R. L., Brotherton, M. S., \& de Kool, M. 1999a, ApJ, 524, 566

Arav, N., Brotherton, M. S., Becker, R. H., Gregg, M. D., White, R. L., Price, T., \& Hack, W. 2001, ApJ, 546, 140

Arav, N., Kaastra, J., Kriss, G. A., Korista, K. T., Gabel, J., \& Proga, D. 2005, ApJ, 620, 665

Arav, N., Korista, K. T., \& de Kool, M. 2002, ApJ, 566, 699

Arav, N., Korista, K. T., de Kool, M., Junkkarinen, V. T., \& Begelman, M. C. 1999b, ApJ, 516, 27

Arav, N., Moe, M., Costantini, E., Korista, K. T., Benn, C., \& Ellison, S. 2008, ApJ, 681, 954

Arnaud, M., \& Evrard, A. E. 1999, MNRAS, 305, 631

Ballero, S. K., Matteucci, F., Ciotti, L., Calura, F., \& Padovani, P. 2008, A\&A, 478, 335

Ballester, P., Grosbol, P., Banse, K., Disaro, A., Dorigo, D., Modigliani, A. Pizarro de la Iglesia, J. A., \& Boitquin, O. 2000, Proc. SPIE, 4010, 246

Bautista, M. A. 2004, A\&A, 420, 763

Bautista, M. A., Peng, J., \& Pradhan, A. K. 1996, ApJ, 460, 372

Bautista, M. A., \& Pradhan, A. K. 1998, ApJ, 492, 650

Blaes, O., Hubeny, I., Agol, E., \& Krolik, J. H. 2001, ApJ, 563, 560

Blandford, R. D., \& Begelman, M. C. 1999, MNRAS, 303, L1

Blum, R. D., \& Pradhan, A. K. 1992, ApJS, 80, 425

Bonning, E. W., Cheng, L., Shields, G. A., Salviander, S., \& Gebhardt, K 2007, ApJ, 659, 211

Brotherton, M. S., Tran, H. D., Becker, R. H., Gregg, M. D., LaurentMuehleisen, S. A., \& White, R. L. 2001, ApJ, 546, 775

de Kool, M., Arav, N., Becker, R. H., Gregg, M. D., White, R. L., LaurentMuehleisen, S. A., Price, T., \& Korista, K. T. 2001, ApJ, 548, 609

de Kool, M., Becker, R. H., Arav, N., Gregg, M. D., \& White, R. L. 2002a, ApJ, 570,514

de Kool, M., Becker, R. H., Gregg, M. D., White, R. L., \& Arav, N. 2002b, ApJ, 567,58

de Kool, M., Korista, K. T., \& Arav, N. 2002c, ApJ, 580, 54

Di Matteo, T., Springel, V., \& Hernquist, L. 2005, Nature, 433, 604

Dufton, P. L., \& Kingston, A. E. 1991, MNRAS, 248, 827

Elvis, M. 2006, Mem. Soc. Astron. Ital., 77, 573

Elvis, M., Marengo, M., \& Karovska, M. 2002, ApJ, 567, L107

Elvis, M., et al. 1994, ApJS, 95, 1

Eriksson, M., Nilsson, H., Veenhuizen, H., \& Long, K. S. 2008, A\&A, 477, 255

Fabian, A. C., Sanders, J. S., Taylor, G. B., Allen, S. W., Crawford, C. S., Johnstone, R. M., \& Iwasawa, K. 2006, MNRAS, 366, 417

Fedchak, J. A., \& Lawler, J. E. 1999, ApJ, 523, 734

Ferland, G. J., Korista, K. T., Verner, D. A., Ferguson, J. W., Kingdon, J. B., \& Verner, E. M. 1998, PASP, 110, 761

Froese Fischer, C. 1983, J. Phys. B: At. Mol. Phys., 16, 157

Fuhr, J., \& Wiese, W. 2006, J. Chem. Ref. Data, 35, 4

Gabel, J. R., et al. 2005, ApJ, 623, 85

Hall, P. B., et al. 2002, ApJS, 141, 267

Hamann, F. W., Barlow, T. A., Chaffee, F. C., Foltz, C. B., \& Weymann, R. J. 2001, ApJ, 550, 142 
Hamann, F., Barlow, T. A., Junkkarinen, V., \& Burbidge, E. M. 1997, ApJ, 478, 80

Hamann, F., \& Ferland, G. 1993, ApJ, 418, 11

Hartman, H., \& Johansson, S. 2000, A\&A, 359, 627

Hamann, F., Korista, K. T., Ferland, G. J., Warner, C., \& Baldwin, J. 2002, ApJ, 564,592

Herbert-Fort, S., Prochaska, J. X., Dessauges-Zavadsky, M., Ellison, S. L., Howk, J. C., Wolfe, A. M., \& Prochter, G. E. 2006, PASP, 118, 1077

Hewett, P. C., \& Foltz, C. B. 2003, AJ, 125, 1784

Hopkins, P. F., Hernquist, L., Cox, T. J., Di Matteo, T., Martini, P., Robertson, B., \& Springel, V. 2005a, ApJ, 630, 705

Hopkins, P. F., Hernquist, L., Martini, P., Cox, T. J., Robertson, B., Di Matteo, T., \& Springel, V. 2005b, ApJ, 625, L71

Hutchings, J. B. 1982, ApJ, 255, 70

Ichikawa, T., van Driel, W., Aoki, T., Soyano, T., Tarusawa, K., \& Yoshida, S. 1994, ApJ, 433, 645

Johansson, S. 1983, MNRAS, 205, 71

Kaiser, N. 1986, MNRAS, 222, 323

Korista, K., Bautista, M., Arav, N., Moe, M., Costantini, E., \& Benn, C. 2008, ApJ, 688, 108

Kurucz, R. L., \& Bell, B. 1995, Atomic line list (Kurucz CD-ROM, Cambridge, MA: Smithsonian Astrophysical Observatory)

Laor, A., Fiore, F., Elvis, M., Wilkes, B. J., \& McDowell, J. C. 1997, ApJ, 477, 93

Lodders, K. 2003, ApJ, 591, 1220

Lucy, L. B. 1995, A\&A, 294, 555

Malkan, M. A. 1983, ApJ, 268, 582

Mathews, W. G., \& Ferland, G. J. 1987, ApJ, 323, 456

McLure, R. J., \& Dunlop, J. S. 2002, MNRAS, 331, 795

Menci, N., Fontana, A., Giallongo, E., Grazian, A., \& Salimbeni, S. 2006, ApJ, 647,753

Michalitsianos, A. G., Kafatos, M., \& Meier, S. R. 1992, ApJ, 389, 649

Moe, M., Arav, N., Bautista, M. A., \& Korista, K. T. 2009, ApJ, 706, 525

Moll, R., et al. 2007, A\&A, 463, 513

Moore, C. E. 1970, Ionization Potentials and Ionization Limits Derived from the Analysis of Optical Spectra (NSRDS-NBS Rep.34; Washington, DC: NBS)

Morris, S. L., Weymann, R. J., Foltz, C. B., Turnshek, D. A., Shectman, S., Price, C., \& Boroson, T. A. 1986, ApJ, 310, 40
Nenkova, M., Sirocky, M. M., Nikutta, R., Ivezić, Ž., \& Elitzur, M. 2008, ApJ, 685,160

Netzer, H., et al. 2003, ApJ, 599, 933

Peterson, B. M. 1997, An Introduction to Active Galactic Nuclei (Cambridge, NY: Cambridge Univ. Press)

Pier, E. A., \& Voit, G. M. 1995, ApJ, 450, 628

Prochter, G. E., Prochaska, J. X., \& Burles, S. M. 2006, ApJ, 639, 766

Quinet, P., Le Dourneuf, M., \& Zeippen, C. J. 1996, A\&AS, 120, 361

Rafferty, D. A., McNamara, B. R., Nulsen, P. E. J., \& Wise, M. W. 2006, ApJ, 652,216

Salpeter, E. E. 1964, ApJ, 140, 796

Savage, B. D., \& Sembach, K. R. 1991, ApJ, 379, 245

Scannapieco, E., \& Oh, S. P. 2004, ApJ, 608, 62

Schlegel, D. J., Finkbeiner, D. P., \& Davis, M. 1998, ApJ, 500, 525

Schneider, D. P., et al. 2003, AJ, 126, 2579

Schneider, D. P., et al. 2007, AJ, 134, 102

Scott, J. E., Kriss, G. A., Brotherton, M., Green, R. F., Hutchings, J., Shull, J. M., \& Zheng, W. 2004a, ApJ, 615, 135

Scott, J. E., et al. 2004b, ApJS, 152, 1

Shakura, N. I., \& Syunyaev, R. A. 1973, A\&A, 24, 337

Shang, Z., et al. 2005, ApJ, 619, 41

Shields, G. A. 1978, Nature, 272, 706

Silk, J., \& Rees, M. J. 1998, A\&A, 331, L1

Springel, V., Di Matteo, T., \& Hernquist, L. 2005, MNRAS, 361, 776

Sternberg, A., Pizzolato, F., \& Soker, N. 2007, ApJ, 656, L5

Tayal, S. S. 2008, ApJS, 179, 534

Telfer, R. C., Zheng, W., Kriss, G. A., \& Davidsen, A. F. 2002, ApJ, 565, 773

Tripp, T. M., Lu, L., \& Savage, B. D. 1996, ApJS, 102, 239

Trump, J. R., et al. 2006, ApJS, 165, 1

Verner, E. M., Verner, D. A., Baldwin, J. A., Ferland, G. J., \& Martin, P. G. 2000, ApJ, 543, 831

Verner, E. M., Verner, D. A., Korista, K. T., Ferguson, J. W., Hamann, F., \& Ferland, G. J. 1999, ApJS, 120, 101

Vreeswijk, P. M., et al. 2007, A\&A, 468, 83

Wampler, E. J., Chugai, N. N., \& Petitjean, P. 1995, ApJ, 443, 586

Zheng, W., Kriss, G. A., Telfer, R. C., Grimes, J. P., \& Davidsen, A. F. 1997, ApJ, 475,469 